\title{
Observer-Based PID Security Control for Discrete Time-Delay Systems under Cyber-Attacks
}

\author{
Di Zhao, Zidong Wang, Daniel W. C. Ho and Guoliang Wei
}

\begin{abstract}
This paper deals with the observer-based proportional-integral-derivative (PID) security control problem for a kind of linear discrete time-delay systems subject to cyberattacks. The cyber-attacks, which include both Denial-of-Service and deception attacks, are allowed to be randomly occurring as regulated by two sequences of Bernoulli distributed random variables with certain probabilities. A novel observer-based PID controller is proposed such that the closed-loop system achieves the desired security level and the quadratic cost criterion (QCC) has an upper bound. Sufficient conditions are derived under which the exponentially mean-square input-to-state stability is guaranteed and the desired security level is then achieved. Subsequently, an upper bound of the QCC is obtained and the explicit expression of the desired PID controller is also parameterized. Finally, the validity of the developed design approach is verified via an illustrative example.
\end{abstract}

Index Terms-Observer-based PID control, security control, Denial-of-Service attacks, deception attacks, exponentially meansquare input-to-state stability.

\section{INTRODUCTION}

$\mathbf{F}$ OR several decades, the proportional-integral-derivative (PID) control scheme has been playing an important role in industry due to its simplicity in controller structure, robustness to external disturbances, and convenience for parameter tuning. The synthesis problem of the PID controller has received particular research interest and a large number of design methods have been developed, see e.g. [1], [9], [15], [46] and the references therein. With the ever-increasing complexity of the controlled plants in the modern industry, conventional PID parameter tuning approaches might not be able to cope with demanding engineering requirements, and much effort has then been devoted to the enhancement of the PID control performance by combining with other advanced control schemes, see e.g. adaptive PID control algorithm [5], intelligent PID control strategy [19], fuzzy PID control method

This work was supported in part by the National Natural Science Foundation of China under Grants 61873148, 61873169 and 61933007, the Research Grants Council of Hong Kong Special Administrative Region under Grant 9042223 and Grant CityU 11200717, and the Alexander von Humboldt Foundation of Germany. (Corresponding author: Guoliang Wei).

D. Zhao is with the Shanghai Key Lab of Modern Optical System, the Department of Control Science and Engineering, University of Shanghai for Science and Technology, Shanghai 200093, China. (Email: zhaodi0907520@163.com)

Z. Wang is with the Department of Computer Science, Brunel University London, Uxbridge, Middlesex, UB8 3PH, United Kingdom. (Email: Zidong.Wang@brunel.ac.uk)

D. W. C. Ho is with the Department of Mathematics, City University of Hong Kong, Kowloon, Hong Kong. (Email: madaniel@cityu.edu.hk)

G. Wei is with the College of Science, University of Shanghai for Science and Technology, Shanghai 200093, China. (Email: guoliang.wei@usst.edu.cn)
[2] and neural network PID control approach [8]. Up to now, the research on the PID control problem has attracted a great deal of attention from the communities of systems science and control engineering.

Most literature concerning PID control has been based on the state feedback. In practice, however, the system states might be immeasurable because of economic constraints or technological limitations, and therefore the observer-based control schemes have become more and more appealing to engineers. For instance, the observer-based control issue has been discussed in [36] for nonlinear stochastic systems in order to achieve the exponential mean-square stability and the variance constraints are also satisfied. The observer-type event-triggered control approach has been presented in [38] to solve the finite-horizon $H_{\infty}$ consensus problem for multiagent systems. In [6], the asynchronous observer-based $H_{\infty}$ controller has been constructed for switched stochastic systems subject to mixed delays, signal quantization as well as packet dropouts. Nevertheless, despite its clear engineering insight, the observer-based PID control problem has not been adequately addressed yet due probably to the observer-induced complexity in parameter tuning, and this situation gives rise to the primary motivation of the present research.

The concept of asymptotic stability has been dominantly used in traditional control theory. In the presence of persistent external inputs including noises/perturbations, asymptotic stability might be inapplicable [10], [16]-[18], and the so-called input-to-state stability (ISS) is well suited to characterize the response of systems to bounded exogenous disturbances. In this regard, many excellent results have been available in the literature. For instance, the input-to-state stabilization problem has been discussed in [20] for a kind of delay differential systems under exogenous disturbances. A matrix inequality approach has been proposed in [14] to analyze the ISS of time-delay systems. In [44], the exponentially meansquare ISS has been addressed for stochastic Cohen-Grossberg neural networks. So far, most ISS-related results have been obtained for continuous-time systems and their discrete-time counterparts have been much fewer due mainly to the lack of appropriate methodologies.

With the quiet revolution of network technologies, the past decade has seen increasing popularity and usage on the networked control systems (NCSs) in practice [21], [34], [37], [39], [42]. However, in comparison with the traditional point-to-point control systems, NCSs are more susceptible to network-induced phenomena including communication delay [7], [26], [27], [32], [43], [47], packet dropout [22], [28], [33], signal quantization [12], fading channel [41]. Furthermore, 
owing to the inherent opening-up characteristic of network links, the information transmission in NCSs is vulnerable to cyber threats which are likely to result in performance degradation or even instability [25], [29], [30]. In this case, the security protection becomes a vitally important issue and the so-called security control problem has received some initial research attention, see [3], [11], [31] and the references therein. Generally speaking, the main idea of the security control is to design a control law such that a desired security level is achieved for the closed-loop system under cyberattacks that include, but are not limited to, Denial-of-Service (DoS) attacks [4], replay attacks [45] and deception attacks [13].

In the context of cyber defence, the attacks initiated by opponents might be unsuccessful in NCSs due to the installed devices or software for security protection. As such, from the defenders' perspective, the cyber-attacks are likely to take place in a random manner and the occurrence mechanism of the attacks can be mathematically modeled by Bernoulli/Markov processes with certain statistical property, see [13], [24] and the references therein. For cyber-physical systems, in addition to the basic security, one would expect that certain system performance can be maintained for necessary system operation. In this case, the quadratic cost behaves as an adequate performance index and a realistic criterion would be to ensure an upper bound on the quadratic cost function for the closed-loop system in spite of the randomly occurring cyber-attacks, which leads to the so-called cost-guaranteed security control [13], [31]. It should be pointed out that, the observer-based PID security control problem for NCSs has not been investigated yet, not to mention the case that the quadratic performance index is also a major concern for system designers. Therefore, the main purpose of this paper is to narrow such a gap.

Summarizing the above discussion, we come to the conclusions that: 1) the observer-based PID control problem is of both theoretical significance and practical importance; 2) the notion of ISS is quite suitable in examining the effect from the cyber-attacks on the system performance; and 3) it is strongly desired to have the cost-guaranteed performance apart from the usual security protection. In view of the above observations, in this paper, we make the first attempt to deal with the PID observer-based control issue for a kind of discrete-time linear systems with time-varying delays under the randomly occurring DoS/deception attacks so as to ensure both the security performance in mean-square sense and the bounded quadratic cost index. It is worth noting that the addressed security control problem is rather challenging due primarily to the analytical complexity resulting from the random occurrence of cyberattacks and the subsequent PID parameter tuning.

The main contributions of this paper are summarized as follows: 1) an observer-based PID controller is, for the first time, constructed for the linear discrete-time system with time-varying delays; 2) the concept of exponentially meansquare ISS is considered in response to randomly occurring DoS/deception attacks; and 3) an upper bound is obtained on the quadratic cost criterion (QCC) of the controlled system in addition to the basic security requirement. The remaining of this paper is organized as follows. Section II formulates the observer-based PID control problem for the linear discretetime systems subject to time-varying delays, where the randomly occurring DoS/deception attacks are introduced in a novel yet unified measurement model. In Section III, the mean-square ISS is first analyzed and sufficient conditions are then derived to ensure both the desired security level and the bounded QCC. A simulation example is shown in Section IV to demonstrate the usefulness and applicability of the controller design algorithm. Finally, we conclude the paper in Section V.

Notation. The notation utilized here is quite normative except where otherwise declared. $\|x\|$ denotes the Euclidean norm of a random variable $x$. The symbol $\otimes$ represents the Kronecker product and the symbol $*$ stands for the ellipsis for symmetric terms. Moreover, we denote the maximum and minimum eigenvalue of matrix $G$ by $\lambda_{\max }(G)$ and $\lambda_{\min }(G)$, respectively.

\section{Problem Formulation And Preliminaries}

Consider the following linear discrete-time system with time delays:

$$
\left\{\begin{aligned}
x(k+1)= & A x(k)+A_{\sigma} x(k-\sigma(k)) \\
& +B u(k)+D w(k) \\
\check{y}(k)= & C x(k) \\
x(l)= & \varphi(l), \quad l=-\sigma_{M}, \cdots,-1,0
\end{aligned}\right.
$$

where $x(k) \in \mathbb{R}^{n_{x}}, u(k) \in \mathbb{R}^{n_{u}}$ and $\check{y}(k) \in \mathbb{R}^{n_{y}}$ represent the system state, the control input and the sensor measurement, respectively. $w(k) \in \mathbb{R}^{n_{w}}$ is the bounded disturbance satisfying

$$
w^{T}(k) w(k) \leqslant \varpi
$$

where $\varpi>0$ is a given scalar. $A, A_{\sigma}, B, C$ and $D$ are known real constant matrices with appropriate dimensions. $\sigma(k)$ is a positive integer that denotes the time-varying delay satisfying

$$
\sigma_{m} \leqslant \sigma(k) \leqslant \sigma_{M}, \quad k \in \mathbb{N}^{+}
$$

where $\sigma_{m}$ and $\sigma_{M}$ are known positive integers. $\varphi(l)(l=$ $\left.-\sigma_{M}, \cdots,-1,0\right)$ are the initial conditions.

In this paper, the data transmission over the measurement channel (sensor-to-observer) is subject to randomly occurring DoS/deception attacks characterized in the following model:

$$
\begin{aligned}
y(k)= & \check{y}(k)+\alpha(k) \beta(k) \mu(k) \\
& +\alpha(k)(1-\beta(k)) v(k)
\end{aligned}
$$

where $y(k)$ is the received signal by the observer subject to attacks, $\mu(k) \in \mathbb{R}^{n_{y}}$ and $v(k) \in \mathbb{R}^{n_{y}}$ stand for, respectively, the deception attack and the DoS attack launched by the attackers described by

$$
\mu(k)=-\check{y}(k)+\varsigma(k)
$$

and

$$
v(k)=-\check{y}(k)
$$


where $\varsigma(k) \neq 0$ denotes an arbitrary bounded signal satisfying

$$
\varsigma^{T}(k) \varsigma(k) \leqslant \zeta
$$

and $\zeta$ is a given positive scalar. The mutually uncorrelated stochastic variables $\alpha(k)$ and $\beta(k)$, which take values on 0 or 1 , are two Bernoulli distributed white sequences with the following probabilities:

$$
\begin{array}{ll}
\operatorname{Prob}\{\alpha(k)=1\}=\bar{\alpha}, & \operatorname{Prob}\{\alpha(k)=0\}=1-\bar{\alpha} \\
\operatorname{Prob}\{\beta(k)=1\}=\bar{\beta}, & \operatorname{Prob}\{\beta(k)=0\}=1-\bar{\beta}
\end{array}
$$

where $\bar{\alpha} \in[0,1)$ and $\bar{\beta} \in[0,1)$ are two known constants.

Remark 1: According to their implementation manners, cyber-attacks can be categorized into three types, i.e., DoS attacks [4], replay attacks [45] and deception attacks [13]. To be specific, DoS attacks occupy a communication channel to prevent the transmission of measurement or control signals. In replay attacks, the effective data transmission is fraudulently or maliciously delayed or repeated. In case of deception attacks, some false information is sent to sensors/controllers so as to degrade or even devastate the performance of the overall systems.

Remark 2: It is worth noticing that the cyber-attacks launched by adversaries might not be always successful due to the defense from the protection institution as well as complicated network environment and, from the defender's perspective, the attacks might occur in a random manner with certain 'success' rate. In this paper, we adopt two sequences of Bernoulli sequences to characterize the random occurrence of the cyber-attacks. In this setup, it is apparent from (4) that the observer receives the normal signals from the sensor when $\alpha(k)=0$. When $\alpha(k)=1$ and $\beta(k)=0$, the data transmission suffers from the DoS attacks and, when $\alpha(k)=1$ and $\beta(k)=1$, the data transmission faces the deception attacks.

To estimate the states of system (1), an observer is constructed in the following form:

$$
\left\{\begin{aligned}
\hat{x}(k+1)= & A \hat{x}(k)+L(y(k)-C \hat{x}(k)) \\
& +A_{\sigma} \hat{x}(k-\sigma(k))+B u(k) \\
\hat{x}(l)= & 0, \quad l=-\sigma_{M}, \cdots,-1,0
\end{aligned}\right.
$$

where $\hat{x}(k) \in \mathbb{R}^{n_{x}}$ is the state estimate and $L$ is the gain matrix of the observer that is to be determined.

In this paper, for the linear discrete-time system (1), we adopt the following observer-based PID control law:

$$
\begin{aligned}
u(k)= & K_{P} \hat{x}(k)+K_{I} \sum_{i=k-d}^{k-1} \hat{x}(i) \\
& +K_{D}(\hat{x}(k)-\hat{x}(k-1))
\end{aligned}
$$

where $K_{P}, K_{I}$ and $K_{D}$ are three PID controller gain matrices to be designed and $d$ is a given scalar representing the time length. For simplicity and without loss of generality, we assume that $d \geqslant \sigma_{M}$.

Remark 3: In the development and application of automatic control, various control algorithms have been proposed to meet the different requirements in practical systems, among which the proportional-integral-derivative (PID) control scheme has proven to be the arguably most widely used one due mainly to the simple structure of the PID controllers. In this paper, we further consider the observer-based PID controller whose structure consists of three loops: the proportional loop (proportional to the state estimation), the integral loop (integral to the state estimation), and the derivative loop (derivative to state estimation). The gain matrices of the above three loops are to be designed so as to meet the specified performance requirements of the closed-loop system. Note that, in the traditional PID controller, the integral loop makes use of all historical information, which incurs huge computational burden. In this paper, the integral operation is set to be performed over a limited but adjustable time length.

Denoting $\tilde{x}(k) \triangleq x(k)-\hat{x}(k)$, the estimation error dynamics is governed by:

$$
\left\{\begin{aligned}
\tilde{x}(k+1)= & (A-L C) \tilde{x}(k)+A_{\sigma} \tilde{x}(k-\sigma(k)) \\
& +D w(k)-L(\alpha(k) \beta(k) \varsigma(k) \\
& -\alpha(k) C x(k)) \\
\tilde{x}(l)= & \varphi(l), \quad l=-\sigma_{M}, \cdots,-1,0
\end{aligned}\right.
$$

where $\tilde{x}(l)$ denotes the initial error.

For the purpose of simplicity, we introduce the following notations:

$$
\eta(k)=\left[\begin{array}{lll}
x^{T}(k) & \tilde{x}^{T}(k)
\end{array}\right]^{T}, \quad \vartheta(k)=\left[\begin{array}{lll}
w^{T}(k) & \varsigma^{T}(k)
\end{array}\right]^{T} .
$$

Implementing the PID control law (9) to system (1), we obtain the closed-loop system as follows:

$$
\left\{\begin{aligned}
\eta(k+1)= & \left(\mathcal{A}_{1}+\tilde{\alpha}(k) \mathcal{A}_{2}\right) \eta(k) \\
& +\mathcal{A}_{\sigma} \eta(k-\sigma(k))+\mathcal{B} \chi(k) \\
& +\left(\mathcal{L}_{1}+\tau(k) \mathcal{L}_{2}\right) \vartheta(k) \\
\eta(l)= & \psi(l), \quad l=-d, \cdots,-1,0
\end{aligned}\right.
$$

where

$$
\begin{aligned}
& \chi(k)=\left[\begin{array}{llll}
\eta^{T}(k-1) & \eta^{T}(k-2) & \cdots & \eta^{T}(k-d)
\end{array}\right]^{T} \\
& \psi(k)=\left[\begin{array}{ll}
\varphi^{T}(k) & 0_{1 \times n_{x}}
\end{array}\right]^{T}, \quad \tilde{\alpha}(k)=\alpha(k)-\bar{\alpha} \\
& \mathcal{A}_{1}^{11}=A+B K_{P}+B K_{D}, \quad \tilde{\beta}(k)=\beta(k)-\bar{\beta} \\
& \mathcal{A}_{1}^{12}=-B\left(K_{D}+K_{P}\right), \quad \mathcal{A}_{1}^{13}=\bar{\alpha} L C \\
& \mathcal{A}_{1}^{14}=A-L C, \quad \tau(k)=\tilde{\alpha}(k) \tilde{\beta}(k)+\tilde{\alpha}(k) \bar{\beta}+\bar{\alpha} \tilde{\beta}(k) \\
& \mathcal{A}_{1}=\left[\begin{array}{cc}
\mathcal{A}_{1}^{11} & \mathcal{A}_{1}^{12} \\
\mathcal{A}_{1}^{13} & \mathcal{A}_{1}^{14}
\end{array}\right], \quad \mathcal{A}_{2}=\left[\begin{array}{cc}
0_{n_{x} \times n_{x}} & 0_{n_{x} \times n_{x}} \\
L C & 0_{n_{x} \times n_{x}}
\end{array}\right] \\
& \mathcal{A}_{\sigma}=\left[\begin{array}{cc}
A_{\sigma} & 0_{n_{x} \times n_{x}} \\
0_{n_{x} \times n_{x}} & A_{\sigma}
\end{array}\right], \quad \mathcal{B}=\left[\begin{array}{c}
B \mathcal{K} \\
0_{n_{x} \times 2 d n_{x}}
\end{array}\right] \\
& \mathcal{L}_{1}=\left[\begin{array}{cc}
D & 0_{n_{x} \times n_{y}} \\
D & -\bar{\alpha} \bar{\beta} L
\end{array}\right], \quad \mathcal{L}_{2}=\left[\begin{array}{cc}
0_{n_{x} \times n_{w}} & 0_{n_{x} \times n_{y}} \\
0_{n_{x} \times n_{w}} & -L
\end{array}\right] \\
& \mathcal{K}=\left[\begin{array}{lllll}
\mathcal{K}_{I}-\mathcal{K}_{D} & \underbrace{\mathcal{K}_{I}}_{d-1} \mathcal{K}_{I} & \cdots & \mathcal{K}_{I}
\end{array}\right] \\
& \mathcal{K}_{I}=\left[\begin{array}{ll}
K_{I} & -K_{I}
\end{array}\right], \quad \mathcal{K}_{D}=\left[\begin{array}{ll}
K_{D} & -K_{D}
\end{array}\right] .
\end{aligned}
$$

It follows from (2) and (7) that

$$
\vartheta^{T}(k) \vartheta(k) \leqslant \delta, \quad \delta=\varpi+\zeta .
$$


Furthermore, the quadratic cost criterion (QCC) associated with (11) is defined as follows:

$$
\begin{aligned}
J(k)= & \limsup _{N \rightarrow \infty} \frac{1}{2 N} \sum_{k=0}^{N} \mathbb{E}\left\{\eta^{T}(k) Q \eta(k)\right. \\
& \left.+u^{T}(k) R u(k)\right\}
\end{aligned}
$$

where $Q$ and $R$ are two given positive-definite weighting matrices.

Before proceeding further, we introduce the following definitions and lemma.

Definition 1: System (11) is said to be exponentially inputto-state stable in mean square if there exist constants $\epsilon>0$, $0<\theta<1$, and $\hbar>0$ such that the system dynamic $\eta(k)$ satisfies

$$
\begin{aligned}
\mathbb{E}\left\{\|\eta(k)\|^{2}\right\} & \leqslant \epsilon \theta^{k} \sup _{l \in[-d, 0]} \mathbb{E}\left\{\|\psi(l)\|^{2}\right\} \\
& +\hbar\|\vartheta(k)\|_{\infty}^{2}, \quad \forall k \geqslant 0
\end{aligned}
$$

where $\|\vartheta(k)\|_{\infty}=\sup _{k}\{\|\vartheta(k)\|\}$.

Definition 2: Let the desired security level be specified as $\varrho>0$. The closed-loop system (11) is said to be $\varrho$-secure in mean-square sense if the inequality $\mathbb{E}\left\{\|\eta(k)\|^{2}\right\} \leqslant \varrho$ holds for all $k \geqslant-d+1$.

Remark 4: It should be pointed out that the concept of input-to-state stability (ISS) presented in Definition 1 is suitable in reflecting the impact from external interferences (e.g. cyber-attacks) on the dynamical performance of the closed-loop system. By resorting to the ISS property, the state trajectory of the closed-loop system could converge to the equilibrium point in the disturbance-free case and could enter a bounded domain under bounded disturbances. Clearly, if (11) is $\varrho$-secure in mean-square sense, then it must be exponentially input-to-state stable in mean square. Here, the parameter $\varrho>0$ indicates the level of the security expressed by the boundedness of the system states.

Lemma 1: (Matrix Inverse Lemma). Let matrices $F, G$, $H$ and $W$ be given with appropriate dimensions. If $F, G$ and $G^{-1}+W F^{-1} H$ are invertible, then the following condition holds

$$
\begin{aligned}
& (F+H G W)^{-1} \\
= & F^{-1}-F^{-1} H\left(G^{-1}+W F^{-1} H\right)^{-1} W F^{-1} .
\end{aligned}
$$

The objective of this paper is to design an observer-based PID controller of the form (9) for system (1) such that the following requirements are met simultaneously:

- Q1) the closed-loop system (11) is $\varrho$-secure in meansquare sense;

- Q2) the QCC (13) has an upper bounded $\bar{J}$.

\section{Main Results}

In this section, we begin with analyzing the security performance of the closed-loop system (11) suffering from cyberattacks. Then, a sufficient condition is established to derive an upper bound for the QCC (13). Finally, the desired observerbased PID controller gain matrices are obtained with the aid of the matrix orthogonal decomposition.

\section{A. Security Analysis}

In this subsection, we shall give a sufficient condition to examine the security performance of the closed-loop system (11). The exponentially mean-square ISS is first analyzed and then a sufficient condition is proposed to ensure the $\varrho$-security of the closed-loop system (11) in mean-square sense.

Theorem 1: Let the positive scalar $\varrho$ and the matrices $K_{P}$, $K_{I}, K_{D}, L$ be given. The closed-loop system (11) is $\varrho$-secure in mean-square sense if there exist positive definite matrices $P_{1}, P_{2}^{s}(s=1,2, \cdots, d), P_{3}$, positive scalars $\varepsilon, \tilde{\kappa}, \tilde{\gamma}$ satisfying

$$
\left\{\begin{array}{l}
\Upsilon_{1}=\left[\begin{array}{ll}
\Upsilon_{1}^{11} & * \\
\Upsilon_{1}^{21} & \Upsilon_{1}^{22}
\end{array}\right]<0 \\
\Upsilon_{2}=\left[\begin{array}{ll}
\Upsilon_{2}^{11} & * \\
\Upsilon_{2}^{21} & \Upsilon_{1}^{22}
\end{array}\right]<0
\end{array}\right.
$$

and

$$
\begin{gathered}
\frac{\tilde{\kappa} \bar{r}-\bar{\rho}_{1}(\bar{r}-1)+\bar{\rho}}{\underline{\rho}} \sup _{l \in[-d, 0]} \mathbb{E}\left\{\|\psi(l)\|^{2}\right\} \\
+\frac{\bar{r} \tilde{\gamma}}{\underline{\rho}(\bar{r}-1)} \delta \leqslant \varrho
\end{gathered}
$$

where

$$
\begin{aligned}
& \Upsilon_{1}^{11}=\operatorname{diag}\left\{\mathcal{P}+\tilde{\kappa} I,-P_{3},-P_{2}\right\} \\
& \Upsilon_{1}^{21}=\left[\begin{array}{ccc}
\sqrt{\tilde{\alpha}} \mathcal{A}_{2} & 0 & 0 \\
\sqrt{\varepsilon} \mathcal{A}_{2} & 0 & 0 \\
\mathcal{A}_{1} & \mathcal{A}_{\sigma} & \mathcal{B} \\
\sqrt{\varepsilon} \mathcal{A}_{1} & \sqrt{\varepsilon} \mathcal{A}_{\sigma} & \sqrt{\varepsilon} \mathcal{B}
\end{array}\right] \\
& \Upsilon_{1}^{22}=-I_{4} \otimes\left(P_{1}^{-1}\right), \quad \Upsilon_{2}^{11}=-\tilde{\gamma} I \\
& \Upsilon_{2}^{21}=\left[\begin{array}{llll}
\mathcal{L}_{1}^{T} & \frac{1}{\sqrt{\varepsilon}} \mathcal{L}_{1}^{T} & \sqrt{\tilde{\tau}} \mathcal{L}_{2}^{T} & \frac{\sqrt{\tilde{\tau}}}{\sqrt{\varepsilon}} \mathcal{L}_{2}^{T}
\end{array}\right]^{T} \\
& \mathcal{P}=-P_{1}+\sum_{s=1}^{d} P_{2}^{s}+\left(\sigma_{M}-\sigma_{m}+1\right) P_{3} \\
& P_{2}=\operatorname{diag}\left\{P_{2}^{1}, P_{2}^{2}, \cdots, P_{2}^{d}\right\}, \check{\alpha}=\bar{\alpha}(1-\bar{\alpha}) \\
& \check{\beta}=\bar{\beta}(1-\bar{\beta}), \quad \check{\tau}=\check{\alpha} \check{\beta}+\check{\alpha} \bar{\beta}^{2}+\check{\beta} \bar{\alpha}^{2} \\
& \underline{\rho}=\min \left\{\lambda_{\min }\left(P_{1}\right), \lambda_{\min }\left(P_{2}\right), \lambda_{\min }\left(P_{3}\right)\right\} \\
& \bar{\rho}_{1}=\lambda_{\max }\left(P_{1}\right), \quad \bar{\rho}_{2}=d \lambda_{\max }\left(P_{2}\right) \\
& \bar{\rho}_{3}=\left(\sigma_{M}-\sigma_{m}+1\right) \lambda_{\max }\left(P_{3}\right), \bar{\rho}=\max \left\{\bar{\rho}_{1}, \bar{\rho}_{2}, \bar{\rho}_{3}\right\}
\end{aligned}
$$

and the constant $\bar{r}>1$ in (17) satisfies

$$
\begin{aligned}
-\tilde{\kappa} \bar{r} & +\bar{\rho}_{1}(\bar{r}-1)+\bar{\rho}_{2} d^{2} \bar{r}^{d}(\bar{r}-1) \\
& +\bar{\rho}_{3} \sigma_{M}\left(\sigma_{M}-\sigma_{m}+1\right) \bar{r}^{\sigma_{M}}(\bar{r}-1)=0 .
\end{aligned}
$$

Proof: In order to analyze the stability of the closed-loop system (11), we select the following Lyapunov-Krasovskii functional:

$$
V(k)=\sum_{i=1}^{4} V_{i}(k)
$$

where

$$
\begin{aligned}
& V_{1}(k)=\eta^{T}(k) P_{1} \eta(k) \\
& V_{2}(k)=\sum_{s=1}^{d} \sum_{q=k-s}^{k-1} \eta^{T}(q) P_{2}^{s} \eta(q)
\end{aligned}
$$


This article has been accepted for publication in a future issue of this journal, but has not been fully edited. Content may change prior to final publication. Citation information: DOI10.1109/TSMC.2019.2952539, IEEE Transactions on Systems, Man, and FINAL

$$
\begin{aligned}
V_{3}(k) & =\sum_{q=k-\sigma(k)}^{k-1} \eta^{T}(q) P_{3} \eta(q) \\
V_{4}(k) & =\sum_{p=k-\sigma_{M}+1}^{k-\sigma_{m}} \sum_{q=p}^{k-1} \eta^{T}(q) P_{3} \eta(q) .
\end{aligned}
$$

Along the trajectory of the closed-loop system (11), we calculate the difference of $V(k)$ and take the mathematical expectation to obtain

$$
\mathbb{E}\{\Delta V(k)\}=\sum_{i=1}^{4} \mathbb{E}\left\{\Delta V_{i}(k)\right\}
$$

where

$$
\begin{aligned}
& \mathbb{E}\left\{\Delta V_{1}(k)\right\} \\
= & \mathbb{E}\left\{V_{1}(k+1)-V_{1}(k)\right\} \\
= & \mathbb{E}\left\{\eta^{T}(k+1) P_{1} \eta(k+1)-\eta^{T}(k) P_{1} \eta(k)\right\} \\
= & \mathbb{E}\left\{\left(\left(\mathcal{A}_{1}+\tilde{\alpha}(k) \mathcal{A}_{2}\right) \eta(k)+\mathcal{A}_{\sigma} \eta(k-\sigma(k))\right.\right. \\
& \left.+\mathcal{B} \chi(k)+\left(\mathcal{L}_{1}+\tau(k) \mathcal{L}_{2}\right) \vartheta(k)\right)^{T} P_{1} \\
& \times\left(\left(\mathcal{A}_{1}+\tilde{\alpha}(k) \mathcal{A}_{2}\right) \eta(k)+\mathcal{A}_{\sigma} \eta(k-\sigma(k))\right. \\
& \left.\left.+\mathcal{B} \chi(k)+\left(\mathcal{L}_{1}+\tau(k) \mathcal{L}_{2}\right) \vartheta(k)\right)-\eta^{T}(k) P_{1} \eta(k)\right\} \\
= & \eta^{T}(k)\left(\mathcal{A}_{1}^{T} P_{1} \mathcal{A}_{1}+\check{\alpha} \mathcal{A}_{2}^{T} P_{1} \mathcal{A}_{2}-P_{1}\right) \eta(k)+\eta^{T}(k \\
& -\sigma(k)) \mathcal{A}_{\sigma}^{T} P_{1} \mathcal{A}_{\sigma} \eta(k-\sigma(k))+\chi^{T}(k) \mathcal{B}^{T} P_{1} \mathcal{B} \chi(k) \\
& +\vartheta^{T}(k)\left(\mathcal{L}_{1}^{T} P_{1} \mathcal{L}_{1}+\check{\tau} \mathcal{L}_{2}^{T} P_{1} \mathcal{L}_{2}\right) \vartheta(k)+2 \eta^{T}(k) \mathcal{A}_{1}^{T} \\
& \times P_{1} \mathcal{A}_{\sigma} \eta(k-\sigma(k))+2 \eta^{T}(k) \mathcal{A}_{1}^{T} P_{1} \mathcal{B} \chi(k)+2 \eta^{T}(k) \\
& \times\left(\mathcal{A}_{1}^{T} P_{1} \mathcal{L}_{1}+\check{\alpha} \bar{\beta} \mathcal{A}_{2}^{T} P_{1} \mathcal{L}_{2}\right) \vartheta(k)+2 \eta^{T}(k-\sigma(k)) \\
& \times \mathcal{A}_{\sigma}^{T} P_{1} \mathcal{B} \chi(k)+2 \eta^{T}(k-\sigma(k)) \mathcal{A}_{\sigma}^{T} P_{1} \mathcal{L}_{1} \vartheta(k) \\
& +2 \chi^{T}(k) \mathcal{B}^{T} P_{1} \mathcal{L}_{1} \vartheta(k)
\end{aligned}
$$

$$
\mathbb{E}\left\{\Delta V_{2}(k)\right\}
$$$$
=\mathbb{E}\left\{V_{2}(k+1)-V_{2}(k)\right\}
$$$$
=\mathbb{E}\left\{\sum_{s=1}^{d}\left(\sum_{q=k+1-s}^{k} \eta^{T}(q) P_{2}^{s} \eta(q)-\sum_{q=k-s}^{k-1} \eta^{T}(q) P_{2}^{s} \eta(q)\right)\right\}
$$$$
=\sum_{s=1}^{d} \mathbb{E}\left\{\eta^{T}(k) P_{2}^{s} \eta(k)-\eta^{T}(k-s) P_{2}^{s} \eta(k-s)\right\}
$$$$
\mathbb{E}\left\{\Delta V_{3}(k)\right\}
$$$$
=\mathbb{E}\left\{V_{3}(k+1)-V_{3}(k)\right\}
$$$$
=\mathbb{E}\left\{\sum_{q=k-\sigma(k+1)+1}^{k} \eta^{T}(q) P_{3} \eta(q)-\sum_{q=k-\sigma(k)}^{k-1} \eta^{T}(q) P_{3} \eta(q)\right\}
$$$$
=\mathbb{E}\left\{\eta^{T}(k) P_{3} \eta(k)-\eta^{T}(k-\sigma(k)) P_{3} \eta(k-\sigma(k))\right.
$$

$$
\left.+\sum_{q=k-\sigma(k+1)+1}^{k-1} \eta^{T}(q) P_{3} \eta(q)-\sum_{q=k-\sigma(k)+1}^{k-1} \eta^{T}(q) P_{3} \eta(q)\right\}
$$$$
=\mathbb{E}\left\{\eta^{T}(k) P_{3} \eta(k)-\eta^{T}(k-\sigma(k)) P_{3} \eta(k-\sigma(k))\right.
$$

$$
\begin{aligned}
& +\sum_{q=k-\sigma_{m}+1}^{k-1} \eta^{T}(q) P_{3} \eta(q)+\sum_{q=k-\sigma(k+1)+1}^{k-\sigma_{m}} \eta^{T}(q) P_{3} \eta(q) \\
& \left.-\sum_{q=k-\sigma(k)+1}^{k-1} \eta^{T}(q) P_{3} \eta(q)\right\} \\
\leqslant & \mathbb{E}\left\{\eta^{T}(k) P_{3} \eta(k)-\eta^{T}(k-\sigma(k)) P_{3} \eta(k-\sigma(k))\right. \\
& \left.+\sum_{i=k-\sigma_{M}+1}^{k-\sigma_{m}} \eta^{T}(q) P_{3} \eta(q)\right\} \\
& \mathbb{E}\left\{\Delta V_{4}(k)\right\} \\
= & \mathbb{E}\left\{V_{4}(k+1)-V_{4}(k)\right\} \\
= & \mathbb{E}\left\{\sum_{p=k-\sigma_{M}+2}^{k-\sigma_{m}+1} \sum_{q=p}^{k} \eta^{T}(q) P_{3} \eta(q)\right. \\
& \left.-\sum_{p=k-\sigma_{M}+1}^{k-\sigma_{m}} \sum_{q=p}^{k} \eta^{T}(q) P_{3} \eta(q)\right\} \\
= & \mathbb{E}\left\{\sum_{p=k-\sigma_{M}+1}^{k-\sigma_{m}}\left(\eta^{T}(k) P_{3} \eta(k)-\eta^{T}(p) P_{3} \eta(p)\right)\right\} \\
= & \mathbb{E}\left\{\left(\sigma_{M}-\sigma_{m}\right) \eta^{T}(k) P_{3} \eta(k)\right. \\
& \left.-\sum_{q=k-\sigma_{M}+1}^{k-\sigma_{m}} \eta^{T}(q) P_{3} \eta(q)\right\} .
\end{aligned}
$$

Substituting (21)-(22) into (20) leads to

$$
\begin{aligned}
& \mathbb{E}\{\Delta V(k)\} \\
\leqslant & \aleph^{T}(k)\left(\Gamma_{1}+\Gamma_{2}\right) \aleph(k)+\check{\alpha} \eta^{T}(k) \mathcal{A}_{2}^{T} P_{1} \mathcal{A}_{2} \eta(k) \\
& +\vartheta^{T}(k)\left(\mathcal{L}_{1}^{T} P_{1} \mathcal{L}_{1}+\check{\tau} \mathcal{L}_{2}^{T} P_{1} \mathcal{L}_{2}\right) \vartheta(k) \\
& +2 \eta^{T}(k)\left(\mathcal{A}_{1}^{T} P_{1} \mathcal{L}_{1}+\check{\alpha} \bar{\beta} \mathcal{A}_{2}^{T} P_{1} \mathcal{L}_{2}\right) \\
& \times \vartheta(k)+2 \eta^{T}(k-\sigma(k)) \mathcal{A}_{d}^{T} P_{1} \mathcal{L}_{1} \vartheta(k) \\
& +2 \chi^{T}(k) \mathcal{B}^{T} P_{1} \mathcal{L}_{1} \vartheta(k) \\
\leqslant & \aleph^{T}(k)\left(\Gamma_{1}+\Gamma_{2}\right) \aleph(k)+\check{\alpha} \eta^{T}(k) \mathcal{A}_{2}^{T} P_{1} \mathcal{A}_{2} \eta(k) \\
& +2 \aleph^{T}(k) \Gamma_{3} \vartheta(k)+\vartheta^{T}(k) \Gamma_{4} \vartheta(k)
\end{aligned}
$$

where

$$
\begin{aligned}
& \aleph(k)=\left[\begin{array}{lll}
\eta^{T}(k) & \eta^{T}(k-\sigma(k)) & \chi^{T}(k)
\end{array}\right]^{T} \\
& \Gamma_{1}=\left[\begin{array}{ccc}
\mathcal{P} & * & * \\
0 & -P_{3} & * \\
0 & 0 & -P_{2}
\end{array}\right] \\
& \Gamma_{2}=\left[\begin{array}{ccc}
\mathcal{A}_{1}^{T} P_{1} \mathcal{A}_{1} & * & * \\
\mathcal{A}_{\sigma}^{T} P_{1} \mathcal{A}_{1} & \mathcal{A}_{\sigma}^{T} P_{1} \mathcal{A}_{\sigma} & * \\
\mathcal{B}^{T} P_{1} \mathcal{A}_{1} & \mathcal{B}^{T} P_{1} \mathcal{A}_{\sigma} & \mathcal{B}^{T} P_{1} \mathcal{B}
\end{array}\right] \\
& \Gamma_{3}=\mathcal{A}^{T} \mathcal{P}_{1} \mathcal{L}, \quad \Gamma_{4}=\mathcal{L}^{T} \mathcal{P}_{1} \mathcal{L}, \quad \mathcal{P}_{1}=I_{2} \otimes P_{1} \\
& \mathcal{A}=\left[\begin{array}{ccc}
\mathcal{A}_{1} & \mathcal{A}_{\sigma} & \mathcal{B} \\
\mathcal{A}_{2} & 0 & 0
\end{array}\right], \quad \mathcal{L}=\left[\begin{array}{c}
\mathcal{L}_{1} \\
\sqrt{\tilde{\tau}} \mathcal{L}_{2}
\end{array}\right] .
\end{aligned}
$$

Applying the elementary inequality $a^{T} P b+b^{T} P a \leqslant$ $\varepsilon a^{T} P a+\frac{1}{\varepsilon} b^{T} P b$ (where $a$ and $b$ are vectors of compatible dimensions) to the term $2 \aleph^{T}(k) \Gamma_{3} \vartheta(k)$, one obtains

$$
2 \aleph^{T}(k) \Gamma_{3} \vartheta(k)
$$




$$
\begin{aligned}
= & 2 \aleph^{T}(k) \mathcal{A}^{T} \mathcal{P}_{1} \mathcal{L} \vartheta(k) \\
= & 2 \eta^{T}(k)\left(\mathcal{A}_{1}^{T} P_{1} \mathcal{L}_{1}+\sqrt{\check{\tau}} \mathcal{A}_{2}^{T} P_{1} \mathcal{L}_{2}\right) \vartheta(k) \\
& +2 \eta^{T}(k-\sigma(k)) \mathcal{A}_{\sigma}^{T} P_{1} \mathcal{L}_{1} \vartheta(k) \\
& +2 \chi^{T}(k) \mathcal{B}^{T} P_{1} \mathcal{L}_{1} \vartheta(k) \\
\leqslant & \varepsilon \aleph^{T}(k) \Gamma_{2} \aleph(k)+\varepsilon \eta^{T}(k) \mathcal{A}_{2}^{T} P_{1} \mathcal{A}_{2} \eta(k) \\
& +\frac{1}{\varepsilon} \vartheta^{T}(k) \Gamma_{4} \vartheta(k) .
\end{aligned}
$$

Subsequently, it implies from (23) and (24) that

$$
\begin{aligned}
& \mathbb{E}\{\Delta V(k)\} \\
\leqslant & \aleph^{T}(k)\left(\Gamma_{1}+\Gamma_{2}\right) \aleph(k)+\check{\alpha} \eta^{T}(k) \mathcal{A}_{2}^{T} P_{1} \mathcal{A}_{2} \eta(k) \\
& +\vartheta^{T}(k) \Gamma_{4} \vartheta(k)+\varepsilon \aleph^{T}(k) \Gamma_{2} \aleph(k)+\varepsilon \eta^{T}(k) \mathcal{A}_{2}^{T} \\
& \times P_{1} \mathcal{A}_{2} \eta(k)+\frac{1}{\varepsilon} \vartheta^{T}(k) \Gamma_{4} \vartheta(k) \\
< & \aleph^{T}(k)\left(\Gamma_{1}+(1+\varepsilon) \Gamma_{2}\right) \aleph(k)+(\varepsilon+\check{\alpha}) \eta^{T}(k) \mathcal{A}_{2}^{T} \\
& \times P_{1} \mathcal{A}_{2} \eta(k)+\left(1+\frac{1}{\varepsilon}\right) \vartheta^{T}(k) \Gamma_{4} \vartheta(k) \\
= & \aleph^{T}(k) \Omega_{1} \aleph(k)+\vartheta^{T}(k) \Omega_{2} \vartheta(k)
\end{aligned}
$$

where

$$
\begin{aligned}
& \Omega_{1}=(1+\varepsilon) \Gamma_{2}+\Omega_{3}, \quad \Omega_{2}=\left(1+\frac{1}{\varepsilon}\right) \Gamma_{4} \\
& \Omega_{3}=\left[\begin{array}{ccc}
(\check{\alpha}+\varepsilon) \mathcal{A}_{2}^{T} P_{1} \mathcal{A}_{2}+\mathcal{P} & 0 & 0 \\
0 & -P_{3} & 0 \\
0 & 0 & -P_{2}
\end{array}\right] .
\end{aligned}
$$

By applying the Schur complement, one has from (16a) and (16b) that

$$
\begin{aligned}
& \mathbb{E}\{\Delta V(k)\} \\
&<\aleph^{T}(k) \Omega_{1} \aleph(k)+\vartheta^{T}(k) \Omega_{2} \vartheta(k) \\
&<-\tilde{\kappa}\|\eta(k)\|^{2}+\tilde{\gamma}\|\vartheta(k)\|_{\infty}^{2} .
\end{aligned}
$$

We are now in a position to proceed with the exponentially ISS analysis of the closed-loop system (11). Based on the definition of $V(k)$, we know that

$$
\begin{gathered}
V(k) \leqslant \bar{\rho}_{1} \mathbb{E}\left\{\|\eta(k)\|^{2}\right\}+\bar{\rho}_{2} \sum_{q=k-d}^{k-1} \mathbb{E}\left\{\|\eta(q)\|^{2}\right\} \\
+\bar{\rho}_{3} \sum_{p=k-\sigma_{M}}^{k-1} \mathbb{E}\left\{\|\eta(p)\|^{2}\right\} .
\end{gathered}
$$

According to the above relation, for any $r>1$, it follows from (26) that

$$
\begin{aligned}
& \mathbb{E}\left\{r^{k+1} V(k+1)\right\}-\mathbb{E}\left\{r^{k} V(k)\right\} \\
= & r^{k+1} \mathbb{E}\{\Delta V(k)\}+r^{k+1} \mathbb{E}\{V(k)\}-r^{k} \mathbb{E}\{V(k)\} \\
< & r^{k+1}\left(-\tilde{\kappa}\|\eta(k)\|^{2}+\tilde{\gamma}\|\vartheta(k)\|_{\infty}^{2}\right) \\
& +r^{k}(r-1) \mathbb{E}\{V(k)\} \\
\leqslant & \pi_{1}(r) r^{k} \mathbb{E}\left\{\|\eta(k)\|^{2}\right\}+\pi_{2}(r) \sum_{q=k-d}^{k-1} r^{k} \mathbb{E}\left\{\|\eta(q)\|^{2}\right\} \\
& +\pi_{3}(r) \sum_{p=k-\sigma_{M}}^{k-1} r^{k} \mathbb{E}\left\{\|\eta(p)\|^{2}\right\}+r^{k+1} \tilde{\gamma}\|\vartheta(k)\|_{\infty}^{2}
\end{aligned}
$$

where

$$
\begin{aligned}
& \pi_{1}(r)=-\tilde{\kappa} r+(r-1) \bar{\rho}_{1} \\
& \pi_{2}(r)=d(r-1) \bar{\rho}_{2} \\
& \left.\pi_{3}(r)=\left(\sigma_{M}-\sigma_{m}+1\right) r-1\right) \bar{\rho}_{3} .
\end{aligned}
$$

Next, for any integer $S \geqslant d+1$, taking summation on both sides of (28) from 0 to $S-1$ with respect to $k$ results in

$$
\begin{aligned}
& \mathbb{E}\left\{r^{S} V(S)\right\}-\mathbb{E}\{V(0)\} \\
< & \pi_{1}(r) \sum_{k=0}^{S-1} r^{k} \mathbb{E}\left\{\|\eta(k)\|^{2}\right\}+\frac{r\left(1-r^{S}\right)}{1-r} \tilde{\gamma}\|\vartheta(k)\|_{\infty}^{2} \\
& +\pi_{2}(r) \sum_{k=0}^{S-1} \sum_{q=k-d}^{k-1} r^{k} \mathbb{E}\left\{\|\eta(q)\|^{2}\right\} \\
& +\pi_{3}(r) \sum_{k=0}^{S-1} \sum_{p=k-\sigma_{M}}^{k-1} r^{k} \mathbb{E}\left\{\|\eta(p)\|^{2}\right\} .
\end{aligned}
$$

The last two items in (29) can be computed as

$$
\begin{aligned}
& \sum_{k=0}^{S-1} \sum_{q=k-d}^{k-1} r^{k} \mathbb{E}\left\{\|\eta(q)\|^{2}\right\} \\
\leqslant & \left(\sum_{q=-d}^{-1} \sum_{k=0}^{q+d}+\sum_{q=0}^{S-d-1} \sum_{k=q+1}^{q+d}\right. \\
& \left.+\sum_{q=S}^{S-1} \sum_{k=q+1}^{S-1}\right) r^{k} \mathbb{E}\left\{\|\eta(q)\|^{2}\right\} \\
\leqslant & d \sum_{q=-d}^{-1} r^{q+d} \mathbb{E}\left\{\|\eta(q)\|^{2}\right\} \\
& +d \sum_{q=0}^{S-d-1} r^{q+d} \mathbb{E}\left\{\|\eta(q)\|^{2}\right\} \\
& +d \sum_{q=S-d}^{S-1} r^{q+d} \mathbb{E}\left\{\|\eta(q)\|^{2}\right\} \\
\leqslant & d r^{d} \sup _{l \in[-d, 0]} \mathbb{E}\left\{\|\psi(l)\|^{2}\right\} \\
& +d r^{d} \sum_{q=0}^{S-1} r^{q} \mathbb{E}\left\{\|\eta(q)\|^{2}\right\}
\end{aligned}
$$

and

$$
\begin{aligned}
& \sum_{k=0}^{S-1} \sum_{q=k-\sigma_{M}}^{k-1} r^{k} \mathbb{E}\left\{\|\eta(q)\|^{2}\right\} \\
& \leqslant\left(\sum_{q=-\sigma_{M}}^{-1} \sum_{k=0}^{q+\sigma_{M}}+\sum_{q=0}^{S-\sigma_{M}-1} \sum_{k=q+1}^{q+\sigma_{M}}\right. \\
& \left.+\sum_{q=S-\sigma_{M}} \sum_{k=q+1}^{S-1}\right) r^{k} \mathbb{E}\left\{\|\eta(q)\|^{2}\right\} \\
& \leqslant \sigma_{M} \sum_{q=-\sigma_{M}}^{-1} r^{q+\sigma_{M}} \mathbb{E}\left\{\|\eta(q)\|^{2}\right\} \\
& \quad+\sigma_{M} \sum_{q=0}^{S-\sigma_{M}-1} r^{q+\sigma_{M}} \mathbb{E}\left\{\|\eta(q)\|^{2}\right\}
\end{aligned}
$$


This article has been accepted for publication in a future issue of this journal, but has not been fully edited. Content may change prior to final publication. Citation information: DOITSMC.2019.2952539, IEEE Transactions on Systems, Man, and Cybernetics: FINAL Systems.

$$
\begin{gathered}
+\sigma_{M} \sum_{q=S-\sigma_{M}}^{S-1} r^{q+\sigma_{M}} \mathbb{E}\left\{\|\eta(q)\|^{2}\right\} \\
\leqslant \sigma_{M} r^{\sigma_{M}} \sup _{l \in\left[-\sigma_{M}, 0\right]} \mathbb{E}\left\{\|\psi(l)\|^{2}\right\} \\
+\sigma_{M} r^{\sigma_{M}} \sum_{q=0}^{S-1} r^{q} \mathbb{E}\left\{\|\eta(q)\|^{2}\right\} .
\end{gathered}
$$

Then, substituting (30)-(31) into (29) yields

$$
\begin{aligned}
& \mathbb{E}\left\{r^{S} V(S)\right\}-\mathbb{E}\{V(0)\} \\
< & \frac{r\left(1-r^{S}\right)}{1-r} \tilde{\gamma}\|\vartheta(k)\|_{\infty}^{2}+p(r) \sum_{k=0}^{S-1} r^{k} \mathbb{E}\left\{\|\eta(k)\|^{2}\right\} \\
& +q(r) \sup _{l \in[-d, 0]} \mathbb{E}\left\{\|\psi(l)\|^{2}\right\}
\end{aligned}
$$

where

$$
\begin{aligned}
& p(r)=\pi_{1}(r)+d r^{d} \pi_{2}(r)+\sigma_{M} r^{\sigma_{M}} \pi_{3}(r) \\
& q(r)=d r^{d} \pi_{2}(r)+\sigma_{M} r^{\sigma_{M}} \pi_{3}(r) .
\end{aligned}
$$

Since $p(1)=-\tilde{\kappa}<0$ and $\lim _{r \rightarrow \infty} p(r)=+\infty$, we know that there exists a scalar $\bar{r}>1$ such that $p_{1}(\bar{r})=0$, which implies that

$$
\begin{aligned}
& \mathbb{E}\left\{\bar{r}^{S} V(S)\right\}-\mathbb{E}\{V(0)\} \\
< & \frac{\bar{r}\left(1-\bar{r}^{S}\right)}{1-\bar{r}} \tilde{\gamma}\|\vartheta(k)\|_{\infty}^{2} \\
& +q(\bar{r}) \sup _{l \in[-d, 0]} \mathbb{E}\left\{\|\psi(l)\|^{2}\right\} .
\end{aligned}
$$

Noting

$$
\mathbb{E}\{V(S)\} \geqslant \underline{\rho} \mathbb{E}\left\{\|\eta(S)\|^{2}\right\}
$$

and

$$
\mathbb{E}\{V(0)\} \leqslant \bar{\rho} \sup _{l \in[-d, 0]} \mathbb{E}\left\{\|\psi(l)\|^{2}\right\}
$$

we obtain

$$
\begin{aligned}
& \mathbb{E}\left\{\|\eta(S)\|^{2}\right\} \\
< & \frac{q(\bar{r})+\bar{\rho}}{\underline{\rho}^{S}} \sup _{l \in[-d, 0]} \mathbb{E}\left\{\|\psi(l)\|^{2}\right\}+\frac{\tilde{\gamma} \bar{r}\left(1-\bar{r}^{S}\right)}{\underline{\rho}^{S}(1-\bar{r})}\|\vartheta(k)\|_{\infty}^{2} \\
< & \frac{q(\bar{r})+\bar{\rho}}{\rho \bar{r}^{S}} \sup _{l \in[-d, 0]} \mathbb{E}\left\{\|\psi(l)\|^{2}\right\}+\frac{\tilde{\gamma} \bar{r}}{\underline{\rho}(\bar{r}-1)}\|\vartheta(k)\|_{\infty}^{2} \\
= & \tilde{\epsilon} \tilde{\theta}^{S} \sup _{l \in[-d, 0]} \mathbb{E}\left\{\|\psi(l)\|^{2}\right\}+\tilde{\hbar}\|\vartheta(k)\|_{\infty}^{2}
\end{aligned}
$$

where $\tilde{\epsilon}=\underline{\rho}^{-1}(q(\bar{r})+\bar{\rho}), \tilde{\theta}=\bar{r}^{-1}$ and $\tilde{\hbar}=\tilde{\gamma} \bar{r}(\underline{\rho}(\bar{r}-1))^{-1}$. This implies that the closed-loop system (11) is exponentially input-to-state stable in the mean square.

Finally, taking (12) into account, it can be found from (17) that

$$
\begin{aligned}
& \mathbb{E}\left\{\|\eta(k)\|^{2}\right\} \\
< & \tilde{\epsilon} \sup _{l \in[-d, 0]} \mathbb{E}\left\{\|\psi(l)\|^{2}\right\}+\tilde{\hbar}\|\vartheta(k)\|_{\infty}^{2} \\
\leqslant & \varrho
\end{aligned}
$$

which means the closed-loop system (11) is $\varrho$-secure. The proof is complete.

\section{B. Guaranteed Cost Analysis}

In this subsection, the analysis on the QCC (13) will be conducted, and an upper bound of the addressed QCC will be provided.

Theorem 2: Let the positive scalar $\varrho$, the positive definite matrices $Q$ and $R$, the matrices $K_{P}, K_{I}, K_{D}$ and $L$ be specified. The closed-loop system (11) is $\varrho$-secure in meansquare sense and the QCC (13) has an upper bound

$$
\bar{J}=\frac{1}{2}\left(\lambda_{\max }(M)+\tilde{\gamma}\right) \delta
$$

if there exist positive definite matrices $P_{1}, P_{2}^{s}(s=$ $1,2, \cdots, d), P_{3}, M$, and positive scalars $\varepsilon, \tilde{\kappa}$ satisfying

$$
\left\{\begin{array}{l}
\Xi_{1}=\left[\begin{array}{ll}
\Upsilon_{1}^{11} & * \\
\Xi_{1}^{21} & \Xi_{1}^{22}
\end{array}\right]<0 \\
\Xi_{2}=\left[\begin{array}{ll}
\Xi_{2}^{11} & * \\
\Xi_{2}^{21} & \Xi_{1}^{22}
\end{array}\right]<0 \\
\Xi_{3}=\left[\begin{array}{ll}
\Xi_{3}^{11} & * \\
\Xi_{3}^{21} & \Xi_{3}^{22}
\end{array}\right]<0 \\
\Xi_{4}=\left[\begin{array}{ll}
\Xi_{1}^{22} & * \\
\Xi_{4}^{21} & \Xi_{2}^{11}
\end{array}\right]<0
\end{array}\right.
$$

and

$$
\begin{gathered}
\frac{\tilde{\kappa} \bar{r}-\bar{\rho}_{1}(\bar{r}-1)+\bar{\rho}}{\underline{\rho}} \sup _{l \in[-d, 0]} \mathbb{E}\left\{\|\psi(l)\|^{2}\right\} \\
+\frac{\bar{r} \tilde{\gamma}}{\underline{\rho}(\bar{r}-1)} \delta \leqslant \varrho
\end{gathered}
$$

where

$$
\begin{aligned}
& \Xi_{1}^{21}=\left[\begin{array}{ccc}
\check{\varepsilon} \mathcal{A}_{2} & 0 & 0 \\
\check{\varepsilon} \mathcal{A}_{1} & \check{\varepsilon} \mathcal{A}_{\sigma} & \check{\varepsilon} \mathcal{B}
\end{array}\right] \\
& \Xi_{1}^{22}=-I_{2} \otimes\left(P_{1}^{-1}\right), \Xi_{2}^{11}=-M, \check{\varepsilon}=\sqrt{1+\varepsilon} \\
& \Xi_{2}^{21}=\mathcal{L}, \Xi_{4}^{21}=\mathcal{L}^{T}, \tilde{\gamma}=\left(1+\varepsilon^{-1}\right) \lambda_{\max }(M) \\
& \Xi_{3}^{11}=\operatorname{diag}\left\{\mathcal{P}+Q-\tilde{\kappa} I,-P_{3},-P_{2}\right\} \\
& \Xi_{3}^{21}=\left[\begin{array}{ccc}
\mathcal{K}_{P}+\mathcal{K}_{D} & 0 & \mathcal{K} \\
\mathcal{A}_{1} & \mathcal{A}_{\sigma} & \mathcal{B} \\
\mathcal{A}_{2} & 0 & 0 \\
0 & 0 & 0
\end{array}\right] \\
& \Xi_{3}^{22}=\left[\begin{array}{cccc}
-R^{-1} & * & * & * \\
0 & -P_{1}^{-1} & * & * \\
0 & 0 & -P_{1}^{-1} & * \\
0 & \mathcal{L}_{1}^{T} & \sqrt{\check{\tau}} \mathcal{L}_{2}^{T} & -M
\end{array}\right]
\end{aligned}
$$

and the constant $\bar{r}>1$ in (39) satisfies

$$
\begin{aligned}
& -\tilde{\kappa} \bar{r}+\bar{\rho}_{1}(\bar{r}-1)+\bar{\rho}_{2} d^{2} \bar{r}^{d}(\bar{r}-1) \\
& \quad+\bar{\rho}_{3} \sigma_{M}\left(\sigma_{M}-\sigma_{m}+1\right) \bar{r}^{\sigma_{M}}(\bar{r}-1)=0 .
\end{aligned}
$$

Proof: The inequality (16a) in Theorem 1 is obtained readily from (38a). In (16b), by selecting $\tilde{\gamma}=(1+$ $\left.\frac{1}{\varepsilon}\right) \lambda_{\max }(M)$, one knows that (16b) holds if (38b) is true. Therefore, the desired security level of the closed-loop system (11) is achieved by (38a)-(38b) and (39).

In what follows, let us investigate the cost functional (13). The combination of the controller (9) and the closed-loop system (11) yields

$$
u(k)=\left(\mathcal{K}_{P}+\mathcal{K}_{D}\right) \eta(k)+\mathcal{K} \chi(k)
$$


where

$$
\mathcal{K}_{P}=\left[\begin{array}{ll}
K_{P} & -K_{P}
\end{array}\right] .
$$

Then, it follows from (23), (26) and (41) that

$$
\begin{aligned}
\mathbb{E} & \left\{2 V(k+1)-2 V(k)+\eta^{T}(k) Q \eta(k)\right. \\
& \left.+u^{T}(k) R u(k)\right\} \\
\leqslant & \mathbb{E}\left\{\Delta V(k)-\tilde{\kappa}\|\eta(k)\|^{2}+\tilde{\gamma}\|\vartheta(k)\|_{\infty}^{2}\right. \\
& \left.+\eta^{T}(k) Q \eta(k)+u^{T}(k) R u(k)\right\} \\
\leqslant & \aleph^{T}(k)\left(\Gamma_{1}+\Gamma_{2}\right) \aleph(k)+\check{\alpha} \eta^{T}(k) \mathcal{A}_{2}^{T} P_{1} \mathcal{A}_{2} \eta(k) \\
& -\tilde{\kappa}\|\eta(k)\|^{2}+2 \aleph^{T}(k) \Gamma_{3} \vartheta(k)+\vartheta^{T}(k) \Gamma_{4} \vartheta(k) \\
& +\tilde{\gamma}\|\vartheta(k)\|_{\infty}^{2}+\eta^{T}(k) Q \eta(k)+\eta^{T}(k)\left(\mathcal{K}_{P}+\mathcal{K}_{D}\right)^{T} \\
& \times R\left(\mathcal{K}_{P}+\mathcal{K}_{D}\right) \eta(k)+2 \eta^{T}(k)\left(\mathcal{K}_{P}+\mathcal{K}_{D}\right)^{T} R \mathcal{K} \chi(k) \\
& +\chi^{T}(k) \mathcal{K}^{T} R \mathcal{K} \chi(k) \\
\leqslant & \aleph^{T}(k)\left(\Gamma_{1}+\mathcal{A}^{T} \mathcal{P}_{1} \mathcal{A}\right) \aleph(k)-\tilde{\kappa}\|\eta(k)\|^{2}+2 \aleph^{T}(k) \Gamma_{3} \\
& \times \vartheta(k)+\vartheta^{T}(k) \Gamma_{4} \vartheta(k)+\tilde{\gamma}\|\vartheta(k)\|_{\infty}^{2}+\eta^{T}(k) Q \eta(k) \\
& +\eta^{T}(k)\left(\mathcal{K}_{P}+\mathcal{K}_{D}\right)^{T} R\left(\mathcal{K}_{P}+\mathcal{K}_{D}\right) \eta(k)+2 \eta^{T}(k) \\
& \times\left(\mathcal{K}_{P}+\mathcal{K}_{D}\right)^{T} R \mathcal{K}_{\chi}(k)+\chi^{T}(k) \mathcal{K}^{T} R \mathcal{K}_{\chi}(k) \\
= & \aleph^{T}(k)\left(\Gamma_{1}+\mathcal{A}^{T} \mathcal{P}_{1} \mathcal{A}\right) \aleph(k)-\tilde{\kappa}\|\eta(k)\|^{2}+2 \aleph^{T}(k) \Gamma_{3} \\
& \times \vartheta(k)+\vartheta^{T}(k)\left(\Gamma_{4}-M\right) \vartheta(k)+\eta^{T}(k)\left(\left(\mathcal{K}_{P}\right.\right. \\
& \left.\left.+\mathcal{K}_{D}\right)^{T} R\left(\mathcal{K}_{P}+\mathcal{K}_{D}\right)+Q\right) \eta(k)+2 \eta^{T}(k)\left(\mathcal{K}_{P}\right. \\
& \left.+\mathcal{K}_{D}\right)^{T} R \mathcal{K}_{\chi}(k)+\chi^{T}(k) \mathcal{K}^{T} R \mathcal{K} \chi(k)+\vartheta^{T}(k) M \\
& \times \vartheta(k)+\tilde{\gamma}\|\vartheta(k)\|_{\infty}^{2} .
\end{aligned}
$$

Taking (12) and (38b) into consideration, one has

$$
\begin{aligned}
& \mathbb{E}\left\{2 V(k+1)-2 V(k)+\eta^{T}(k) Q \eta(k)\right. \\
& \left.+u^{T}(k) R u(k)\right\} \\
\leqslant & \aleph^{T}(k)\left(\Gamma_{1}+\mathcal{A}^{T} \mathcal{P}_{1} \mathcal{A}\right) \aleph(k)-\tilde{\kappa}\|\eta(k)\|^{2}+\eta^{T}(k) \\
& \times\left(\left(\mathcal{K}_{P}+\mathcal{K}_{D}\right)^{T} R\left(\mathcal{K}_{P}+\mathcal{K}_{D}\right)+Q\right) \eta(k) \\
& +2 \eta^{T}(k)\left(\mathcal{K}_{P}+\mathcal{K}_{D}\right)^{T} R \mathcal{K} \chi(k)+\chi^{T}(k) \mathcal{K}^{T} R \mathcal{K} \\
& \times \chi(k)+2 \aleph^{T}(k) \Gamma_{3} \vartheta(k)+\vartheta^{T}(k)\left(\Gamma_{4}-M\right) \vartheta(k) \\
& +\left(\lambda_{\max }(M)+\tilde{\gamma}\right) \delta \\
= & \aleph^{T}(k) \Pi_{1} \aleph(k)+2 \aleph^{T}(k) \Gamma_{3} \vartheta(k)-\vartheta^{T}(k)\left(M-\Gamma_{4}\right) \\
& \times \vartheta(k)+\left(\lambda_{\max }(M)+\tilde{\gamma}\right) \delta \\
\leqslant & \aleph^{T}(k)\left(\Pi_{1}+\Pi_{2}\right) \aleph(k)+\left(\lambda_{\max }(M)+\tilde{\gamma}\right) \delta
\end{aligned}
$$

where

$$
\begin{aligned}
& \Pi_{0}=\left[\begin{array}{c}
-\tilde{\kappa} I+\left(\mathcal{K}_{P}+\mathcal{K}_{D}\right)^{T} R\left(\mathcal{K}_{P}+\mathcal{K}_{D}\right)+Q \\
0 \\
\mathcal{K}^{T} R\left(\mathcal{K}_{P}+\mathcal{K}_{D}\right)
\end{array}\right. \\
& \left.\begin{array}{cc}
0 & \left(\mathcal{K}_{P}+\mathcal{K}_{D}\right)^{T} R \mathcal{K} \\
0 & 0 \\
0 & \mathcal{K}^{T} R \mathcal{K}
\end{array}\right] \\
& \Pi_{1}=\Gamma_{1}+\mathcal{A}^{T} \mathcal{P}_{1} \mathcal{A}+\Pi_{0} \\
& \Pi_{2}=\Gamma_{3}\left(M-\Gamma_{4}\right)^{-1} \Gamma_{3}^{T} .
\end{aligned}
$$

In light of (38d) and Lemma 1, we obtain that

$$
\Pi_{2}=\Gamma_{3}\left(M-\Gamma_{4}\right)^{-1} \Gamma_{3}^{T}
$$

$$
\begin{aligned}
& =\mathcal{A}^{T} \mathcal{P}_{1} \mathcal{L}\left(M-\Gamma_{4}\right)^{-1} \mathcal{L}^{T} \mathcal{P}_{1} \mathcal{A} \\
& =\mathcal{A}^{T} \mathcal{P}_{1} \mathcal{L}\left(M-\mathcal{L}^{T} \mathcal{P}_{1} \mathcal{L}\right)^{-1} \mathcal{L}^{T} \mathcal{P}_{1} \mathcal{A} \\
& =\mathcal{A}^{T}\left(\mathcal{P}_{1}^{-1}-\mathcal{L} M^{-1} \mathcal{L}^{T}\right)^{-1} \mathcal{A}-\mathcal{A}^{T} \mathcal{P}_{1} \mathcal{A}
\end{aligned}
$$

and, furthermore, it follows from (43) and (44) that

$$
\begin{aligned}
& \mathbb{E}\left\{2 V(k+1)-2 V(k)+\eta^{T}(k) Q \eta(k)\right. \\
&\left.+u^{T}(k) R u(k)\right\} \\
& \leqslant \aleph^{T}(k) \Pi_{3} \aleph(k)+\left(\lambda_{\max }(M)+\tilde{\gamma}\right) \delta
\end{aligned}
$$

where

$$
\Pi_{3}=\Gamma_{1}+\Pi_{0}+\mathcal{A}^{T}\left(\mathcal{P}_{1}^{-1}-\mathcal{L} M^{-1} \mathcal{L}^{T}\right)^{-1} \mathcal{A} .
$$

Next, by exploiting the Schur Complement Lemma, we conclude from (38c) that $\Pi_{3}<0$, which results in

$$
\begin{aligned}
& \sup \sum_{k=0}^{N} \mathbb{E}\left\{\eta^{T}(k) Q \eta(k)+u^{T}(k) R u(k)\right\} \\
\leqslant & \sup \sum_{k=0}^{N} \mathbb{E}\left\{\aleph^{T}(k) \Pi_{3} \aleph(k)+\left(\lambda_{\max }(M)+\tilde{\gamma}\right) \delta\right. \\
& -2 \mathbb{E}\{V(k+1)-V(k)\}\} \\
\leqslant & 2 V(0)+(N+1)\left(\lambda_{\max }(M)+\tilde{\gamma}\right) \delta \\
& -2 \mathbb{E}\{V(N+1)\} \\
\leqslant & 2 V(0)+(N+1)\left(\lambda_{\max }(M)+\tilde{\gamma}\right) \delta .
\end{aligned}
$$

Finally, it implies from (13) and (46) that

$$
\begin{aligned}
J(k) & =\limsup _{N \rightarrow \infty} \frac{1}{2 N} \sum_{k=0}^{N} \mathbb{E}\left\{\eta^{T}(k) Q \eta(k)+u^{T}(k) R u(k)\right\} \\
& \leqslant \limsup _{N \rightarrow \infty} \frac{1}{2 N}\left(2 V(0)+(N+1)\left(\lambda_{\max }(M)+\tilde{\gamma}\right) \delta\right) \\
& =\frac{\left(\lambda_{\max }(M)+\tilde{\gamma}\right) \delta}{2}
\end{aligned}
$$

which completes the proof.

\section{Observer-Based PID Controller Design}

In this subsection, an observer-based PID control law will be designed for system (1) such that the closed-loop system (11) is $\varrho$-secure in mean-square sense and the addressed QCC (13) has an upper bound. It will be shown that the gain matrices of the desired controller can be acquired via solving a set of linear matrix inequalities (LMIs).

Theorem 3: Let the positive definite matrices $Q, R$ and the positive scalar $\varrho$ be specified. The closed-loop system (11) is $\varrho$-secure in mean-square sense and the QCC (13) has the upper bound $\bar{J}=\frac{1}{2}\left(\lambda_{\min }^{-1}(\hat{M})+\tilde{\gamma}\right) \delta$ if there exist positive definite matrices $\hat{P}_{1}, P_{2}^{s}(s=1,2, \cdots, d), P_{3}, \hat{M}$, positive scalars $\tilde{\kappa}, \tilde{\varepsilon}>1$, and matrices $\Lambda_{11}, \Lambda_{12}, \Lambda_{22}, \check{K}_{P}, \check{K}_{I}, \check{K}_{D}$ and $\check{L}$ 
satisfying

$$
\left\{\begin{array}{l}
\tilde{\Xi}_{1}=\left[\begin{array}{ll}
\Upsilon_{1}^{11} & * \\
\tilde{\Xi}_{1}^{21} & \tilde{\Xi}_{1}^{22}
\end{array}\right]<0 \\
\tilde{\Xi}_{2}=\left[\begin{array}{ll}
\tilde{\Xi}_{2}^{11} & * \\
\tilde{\Xi}_{2}^{21} & \tilde{\Xi}_{2}^{22}
\end{array}\right]<0 \\
\tilde{\Xi}_{3}=\left[\begin{array}{cc}
\Xi_{3}^{11} & * \\
\tilde{\Xi}_{3}^{21} & \tilde{\Xi}_{3}^{22}
\end{array}\right]<0 \\
\tilde{\Xi}_{4}=\left[\begin{array}{ll}
\tilde{\Xi}_{2}^{22} & * \\
\tilde{\Xi}_{4}^{21} & \Xi_{2}^{11}
\end{array}\right]<0
\end{array}\right.
$$

and

$$
\begin{gathered}
\frac{\tilde{\kappa} \bar{r}-\bar{\rho}_{1}(\bar{r}-1)+\bar{\rho}}{\underline{\rho}} \sup _{l \in[-d, 0]} \mathbb{E}\left\{\|\psi(l)\|^{2}\right\} \\
+\frac{\bar{r} \tilde{\gamma}}{\underline{\rho}(\bar{r}-1)} \delta \leqslant \varrho
\end{gathered}
$$

where

$$
\begin{aligned}
& \tilde{\Xi}_{1}^{21}=\left[\begin{array}{ccc}
0 & 0 & 0 \\
\check{\varepsilon} \Sigma_{21} & 0 & 0 \\
\check{\varepsilon} \Sigma_{31} & \check{\varepsilon} \Sigma_{32} & \check{\varepsilon} \Sigma_{33} \\
\check{\varepsilon} \Sigma_{41} & \check{\varepsilon} \Sigma_{42} & 0
\end{array}\right] \\
& \tilde{\Xi}_{1}^{22}=\operatorname{diag}\left\{-\hat{P}_{1},-\hat{P}_{1}, \tilde{P}_{1},-\hat{P}_{1}\right\} \\
& \tilde{\Xi}_{2}^{11}=-\hat{M}, \quad \tilde{\Xi}_{2}^{21}=\left[\begin{array}{c}
\check{\mathcal{L}}_{1} \\
\sqrt{\tilde{\tau}} \check{\mathcal{L}}_{2}
\end{array}\right] \\
& \tilde{\Xi}_{2}^{22}=-\mathcal{P}_{1}, \quad \tilde{\Xi}_{4}^{21}=\left[\begin{array}{ll}
\check{\mathcal{L}}_{1}^{T} & \sqrt{\check{\tau}} \check{\mathcal{L}}_{2}^{T}
\end{array}\right] \\
& \tilde{\Xi}_{3}^{21}=\left[\begin{array}{ccc}
\check{\mathcal{K}}_{P}+\check{\mathcal{K}}_{D} & 0 & \check{\mathcal{K}} \\
\Sigma_{31} & \Sigma_{32} & \Sigma_{33} \\
\Sigma_{41} & \Sigma_{42} & 0 \\
0 & 0 & 0 \\
\Sigma_{21} & 0 & 0 \\
0 & 0 & 0
\end{array}\right] \\
& \tilde{\Xi}_{3}^{22}=\left[\begin{array}{cccccc}
\tilde{R} & * & * & * & * & * \\
0 & \tilde{P}_{1} & * & * & * & * \\
0 & 0 & -\hat{P}_{1} & * & * & * \\
0 & 0 & 0 & -\hat{P}_{1} & * & * \\
0 & 0 & 0 & 0 & -\hat{P}_{1} & * \\
0 & \Sigma_{62} & \Sigma_{63} & 0 & \sqrt{\sigma^{*}} \Sigma_{65} & -\tilde{M}
\end{array}\right] \\
& \tilde{\gamma}=\frac{\check{\varepsilon}^{2}}{\lambda_{\min }(\hat{M})\left(\check{\varepsilon}^{2}-1\right)}, \quad \Sigma_{21}=\left[\begin{array}{ll}
\check{L} C & 0
\end{array}\right] \\
& \Sigma_{31}=\left[\Lambda \Theta A+\bar{K}_{P}+\bar{K}_{D}-\bar{K}_{P}-\bar{K}_{D}\right] \\
& \Sigma_{32}=\left[\begin{array}{ll}
\Lambda \Theta A_{\sigma} & 0
\end{array}\right], \quad \Sigma_{42}=\left[\begin{array}{ll}
\hat{P}_{1} A_{\sigma} & 0
\end{array}\right] \\
& \Sigma_{41}=\left[\begin{array}{ll}
\bar{\alpha} \check{L} C & \hat{P}_{1} A-\check{L} C
\end{array}\right], \quad \Sigma_{33}=\overline{\mathcal{K}} \\
& \Sigma_{62}=\left[\begin{array}{c}
\hat{P}_{1} D \\
0
\end{array}\right], \quad \Sigma_{63}=\left[\begin{array}{c}
\hat{P}_{1} D \\
-\bar{\alpha} \bar{\beta} \hat{L}
\end{array}\right] \\
& \Sigma_{65}=\left[\begin{array}{c}
0 \\
-\check{L}
\end{array}\right], \quad \tilde{M}=M-2 \hat{P}_{1} \\
& \check{\mathcal{K}}_{P}=\left[\begin{array}{ll}
\check{K}_{P} & -\check{K}_{P}
\end{array}\right], \quad \check{\mathcal{K}}_{I}=\left[\begin{array}{ll}
\check{K}_{I} & -\check{K}_{I}
\end{array}\right] \\
& \check{\mathcal{K}}_{D}=\left[\begin{array}{ll}
\check{K}_{D} & -\check{K}_{D}
\end{array}\right], \quad \bar{K}_{P}=\left[\begin{array}{ll}
\check{K}_{P}^{T} & 0
\end{array}\right]^{T} \\
& \bar{K}_{I}=\left[\begin{array}{cc}
\check{K}_{I}^{T} & 0
\end{array}\right]^{T}, \quad \bar{K}_{D}=\left[\begin{array}{cc}
\check{K}_{D}^{T} & 0
\end{array}\right]^{T}
\end{aligned}
$$

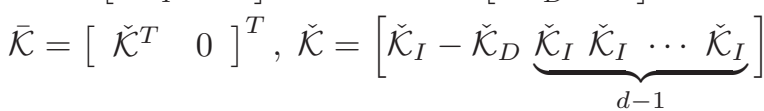

$$
\begin{aligned}
& \check{\mathcal{L}}_{1}=\left[\begin{array}{cc}
\hat{P}_{1} D & 0 \\
\hat{P}_{1} D & -\bar{\alpha} \bar{\beta} \check{L}
\end{array}\right], \quad \hat{\mathcal{L}}_{2}=\left[\begin{array}{cc}
0 & 0 \\
0 & -\check{L}
\end{array}\right] \\
& \Lambda=\left[\begin{array}{cc}
\Lambda_{11} & \Lambda_{12} \\
0 & \Lambda_{22}
\end{array}\right], \quad \tilde{R}=R-\Lambda_{11}-\Lambda_{11}^{T} \\
& \tilde{P}_{1}=\hat{P}_{1}-\Lambda \Theta-\Theta^{T} \Lambda^{T}, \quad P_{1}=I_{2} \otimes \hat{P}_{1} \\
& \Theta=\left[B\left(B^{T} B\right)^{-1}\left(B^{T}\right)^{\perp}\right]^{T}
\end{aligned}
$$

and the constant $\bar{r}>1$ in (49) is the solution to the following equation

$$
\begin{aligned}
& -\tilde{\kappa} \bar{r}+\bar{\rho}_{1}(\bar{r}-1)+\bar{\rho}_{2} d^{2} \bar{r}^{d}(\bar{r}-1) \\
& \quad+\bar{\rho}_{3} \sigma_{M}\left(\sigma_{M}-\sigma_{m}+1\right) \bar{r}^{\sigma_{M}}(\bar{r}-1)=0 .
\end{aligned}
$$

In addition, the gain matrices of the observer (8) and the PID controller (9) are given by

$$
\begin{array}{ll}
K_{P}=\Lambda_{11}^{-1} \check{K}_{P}, \quad K_{I}=\Lambda_{11}^{-1} \check{K}_{I} \\
K_{D}=\Lambda_{11}^{-1} \check{K}_{D}, \quad L=\hat{P}_{1}^{-1} \check{L} .
\end{array}
$$

Proof: Letting $\hat{M}=M^{-1}$, we perform the congruence transformation to the inequality (38b) by $\operatorname{diag}\left\{\hat{M}, P_{1}, P_{1}\right\}$ and to the inequality (38d) by $\operatorname{diag}\left\{P_{1}, P_{1}, \hat{M}\right\}$, respectively. Then, we arrive at (48b) and (48d). Furthermore, by pre- and post-multiplying the inequality (38a) by $\operatorname{diag}\left\{I, I, I, \hat{P}_{1}, \hat{P}_{1}, \Lambda \Theta, \hat{P}_{1}\right\}$ and its transposition, we have

$$
\bar{\Xi}_{1}=\left[\begin{array}{cc}
\Upsilon_{1}^{11} & * \\
\Xi_{1}^{21} & \Xi_{1}^{22}
\end{array}\right]<0
$$

where

$$
\begin{aligned}
& \bar{\Xi}_{1}^{21}=\left[\begin{array}{ccc}
0 & 0 & 0 \\
\check{\varepsilon} \bar{\Sigma}_{21} & 0 & 0 \\
\check{\varepsilon} \bar{\Sigma}_{31} & \check{\varepsilon} \Sigma_{32} & \check{\varepsilon} \bar{\Sigma}_{33} \\
\check{\varepsilon} \bar{\Sigma}_{41} & \check{\varepsilon} \Sigma_{42} & 0
\end{array}\right] \\
& \bar{\Xi}_{1}^{22}=\operatorname{diag}\left\{-\hat{P}_{1},-\hat{P}_{1},-\Lambda \Theta \hat{P}_{1}^{-1} \Theta^{T} \Lambda^{T},-\hat{P}_{1}\right\} \\
& \bar{\Sigma}_{21}=\left[\begin{array}{cc}
\hat{P}_{1} L C & 0
\end{array}\right], \bar{\Sigma}_{33}=\Lambda \Theta B \mathcal{K} \\
& \bar{\Sigma}_{31}=\left[\begin{array}{c}
\left(\Lambda \Theta A+\Lambda \Theta B\left(K_{P}+K_{D}\right)\right)^{T} \\
-\left(\Lambda \Theta B\left(K_{P}+K_{D}\right)\right)^{T}
\end{array}\right]^{T} \\
& \bar{\Sigma}_{41}=\left[\begin{array}{cc}
\bar{\alpha} \hat{P}_{1} L C & \hat{P}_{1} A-\hat{P}_{1} L C
\end{array}\right] .
\end{aligned}
$$

Similarly, pre- and post-multiplying the inequality (38c) by $\operatorname{diag}\left\{I, I, I, \Lambda_{11}, \Lambda \Theta, \hat{P}_{1}, \hat{P}_{1}, \hat{P}_{1}, \hat{P}_{1}\right\}$ and its transposition, one has

$$
\bar{\Xi}_{3}=\left[\begin{array}{cc}
\Xi_{3}^{11} & * \\
\Xi_{3}^{21} & \Xi_{3}^{22}
\end{array}\right]<0
$$

where

$$
\begin{aligned}
\bar{\Xi}_{3}^{21} & =\left[\begin{array}{ccc}
\Lambda_{11} \mathcal{K}_{P}+\Lambda_{11} \mathcal{K}_{D} & 0 & \Lambda_{11} \mathcal{K} \\
\bar{\Sigma}_{31} & \Sigma_{32} & \bar{\Sigma}_{33} \\
\bar{\Sigma}_{41} & \Sigma_{42} & 0 \\
0 & 0 & 0 \\
\bar{\Sigma}_{21} & 0 & 0 \\
0 & 0 & 0
\end{array}\right] \\
\bar{\Xi}_{3}^{22} & =\left[\begin{array}{cc}
-\Lambda_{11} R^{-1} \Lambda_{11}^{T} & * \\
0 & -\Lambda \Theta \hat{P}_{1}^{-1} \Theta^{T} \Lambda^{T} \\
0 & 0 \\
0 & 0 \\
0 & 0 \\
0 & \Sigma_{62}
\end{array}\right.
\end{aligned}
$$


This article has been accepted for publication in a future issue of this journal, but has not been fully edited. Content may change prior to final publication. Citation information: DOI10.1109/TSMC.2019.2952539, IEEE Transactions on Systems, Man, and

$$
\begin{aligned}
& \left.\begin{array}{cccc}
* & * & * & \\
* & * & * & * \\
-\hat{P}_{1} & * & * & * \\
0 & -\hat{P}_{1} & * & * \\
0 & 0 & -\hat{P}_{1} & * \\
\bar{\Sigma}_{63} & 0 & \sqrt{\sigma^{*} \bar{\Sigma}_{65}} & -\hat{P}_{1} M \hat{P}_{1}
\end{array}\right] \\
& \bar{\Sigma}_{63}=\left[\begin{array}{ll}
\hat{P}_{1} D & -\bar{\alpha} \bar{\beta} \hat{P}_{1} L
\end{array}\right], \bar{\Sigma}_{65}=\left[\begin{array}{ll}
0 & -\hat{P}_{1} L
\end{array}\right] .
\end{aligned}
$$

It is clear that

$$
\begin{aligned}
& \Lambda \Theta+\Theta^{T} \Lambda^{T}-\Lambda \Theta \hat{P}_{1}^{-1} \Theta^{T} \Lambda^{T}-\hat{P}_{1} \\
= & -\left(\Lambda \Theta-\hat{P}_{1}\right) \hat{P}_{1}^{-1}\left(\Lambda \Theta-\hat{P}_{1}\right)^{T} \leqslant 0 \\
& \Lambda_{11}+\Lambda_{11}^{T}-\Lambda_{11} R^{-1} \Lambda_{11}^{T}-R \\
= & -\left(\Lambda_{11}-R\right) R^{-1}\left(\Lambda_{11}-R\right)^{T} \leqslant 0 \\
& \hat{P}_{1}+\hat{P}_{1}^{T}-\hat{P}_{1} M \hat{P}_{1}^{T}-M^{-1} \\
= & -\left(\hat{P}_{1}-M^{-1}\right) M\left(\hat{P}_{1}-M^{-1}\right)^{T} \leqslant 0,
\end{aligned}
$$

which implies that

$$
\begin{aligned}
-\Lambda \Theta \hat{P}_{1}^{-1} \Theta^{T} \Lambda^{T} & \leqslant \hat{P}_{1}-\Lambda \Theta-\Theta^{T} \Lambda^{T} \\
-\Lambda_{11} R^{-1} \Lambda_{11}^{T} & \leqslant R-\Lambda_{11}-\Lambda_{11}^{T} \\
-\hat{P}_{1} M \hat{P}_{1}^{T} & \leqslant \hat{M}-2 \hat{P}_{1} .
\end{aligned}
$$

Taking (54) into consideration and utilizing the variable substitution

$$
\begin{array}{ll}
\check{K}_{P}=\Lambda_{11} K_{P}, \quad \check{K}_{I}=\Lambda_{11} K_{I} \\
\check{K}_{D}=\Lambda_{11} K_{D}, \quad \check{L}=\hat{P}_{1} L
\end{array}
$$

we conclude that (51) is ensured by (48a), and (52) is ensured by $(48 \mathrm{c})$. Therefore, the rest of the proof follows immediately from Theorem 2 .

Remark 5: In this paper, by employing the orthogonal decomposition, we introduce a free matrix $\Lambda$ with an unique structure and construct a matrix $\Theta=\left[B\left(B^{T} B\right)^{-1}\left(B^{T}\right)^{\perp}\right]^{T}$ in order to cope with the coupling terms $P B K_{P}, P B K_{I}$ and $P B K_{D}$ in Theorem 2. It is clear that, when the parameter $\check{\varepsilon}$ is fixed, inequalities (48a)-(48d) will be simplified to linear matrix inequalities (LMIs) that are easy to solve by using standard computational packages. Moreover, the introduction of parameter $\check{\varepsilon}$ provides additional flexibility for designing the controller, which is also beneficial for improving the security level of the system.

Remark 6: Up to now, the observer-based PID control problem has been tackled for a kind of linear discrete-time systems under random occurrence of cyber-attacks. By resorting to the designed controller, the security requirement has been ensured with a guaranteed upper bound on the QCC. Existence conditions for the desired controller have been proposed by means of the LMI-based orthogonal decomposition approach. The developed methodology has the potential to design more advanced control schemes, e.g. adaptive PID control algorithm, intelligent PID control strategy, fuzzy PID control method and neural network PID control approach. The main results established in Theorems 1-3 distinguish themselves from existing literature in the following three aspects: 1) the problem addressed is new in the sense that the observer-based PID control problem is, for the first time, designed for discrete-time system with time-varying delays under cyber-attacks; 2) the concept of $\varrho$-security in meansquare is new, which is well placed to describe the security level (size of the boundedness) of the system states in response to randomly occurring DoS/deception attacks; and 3) the performance evaluation is new, which provides an upper bound on the quadratic cost criterion, thereby further improving the closed-loop performance apart from the fundamental security requirement.

\section{ILLUSTRATIVE EXAMPLE}

Consider the observer-based PID security control problem for a geared DC motor, which is a component of the MS150 Modular Servo System [40]. With a sampling period of $0.01 \mathrm{~s}$, the discrete-time model is obtained with the following system matrix and measurement matrix:

$$
A=\left[\begin{array}{ll}
1 & 0.0098 \\
0 & 0.9653
\end{array}\right], \quad C=\left[\begin{array}{l}
1 \\
0
\end{array}\right]^{T} .
$$

In the MS150 Modular Servo System, the input $u(k)$ is a voltage and the output $\check{y}(k)$ is a rotary angle of the extended shaft, which is also called the position of the shaft. Furthermore, other parameters are presented as follows:

$$
A_{\sigma}=\left[\begin{array}{cc}
0.03 & 0 \\
0 & 0.02
\end{array}\right], B=\left[\begin{array}{c}
0.729 \\
0.21
\end{array}\right], D=\left[\begin{array}{l}
0.65 \\
0.71
\end{array}\right]^{T} \text {. }
$$

\section{A. Effectiveness of the Developed Observer-Based PID Con- trol Scheme}

In this example, the success probabilities of the cyberattacks are assumed to be $\bar{\alpha}=0.7$ and $\bar{\beta}=0.5$. Moreover, the time span of the integral loop in PID controller (9) is taken as $d=3$, the lower and upper bounds on the timevarying delay are taken as $\sigma_{m}=1$ and $\sigma_{M}=3$, respectively. Furthermore, the exogenous disturbances are selected to be $\omega(k)=0.25 \cos (k)$ and $\varsigma(k)=0.25 \sin (k)$. Set the security parameter $\varrho=0.29$, the bound on disturbance input $\delta=0.5$, and the weighting matrices $Q=0.05 I$ and $R=0.05 I$. Choose the initial values of the state as $x(-3)=x(-2)=x(-1)=$ $x(0)=\left[\begin{array}{ll}-0.8 & 0.8\end{array}\right]^{T}$.

By means of the Matlab software (with the YALMIP 3.0), the solution to matrix inequalities (48a)-(48d) in Theorem 3 is computed as follows:

$$
\begin{aligned}
& \hat{P}_{1}= {\left[\begin{array}{ccc}
86.0357 & -31.8339 \\
-31.8339 & 56.6393
\end{array}\right], \check{L}=\left[\begin{array}{c}
31.2034 \\
-0.2381
\end{array}\right] } \\
& P_{2}^{1}= {\left[\begin{array}{cccc}
99.3003 & -0.9063 & -6.1332 & 0.3238 \\
-0.9063 & 101.7703 & 0.1872 & -0.0174 \\
-6.1332 & 0.1872 & 100.1997 & 0.3192 \\
0.3238 & -0.0174 & 0.3192 & 102.0761
\end{array}\right] } \\
& P_{2}^{2}= {\left[\begin{array}{cccc}
99.3003 & -0.9063 & -6.1332 & 0.3238 \\
-0.9063 & 101.7703 & 0.1872 & -0.0174 \\
-6.1332 & 0.1872 & 100.1997 & 0.3192 \\
0.3238 & -0.0174 & 0.3192 & 102.0761
\end{array}\right] } \\
& P_{2}^{3}= \\
& {\left[\begin{array}{cccc}
99.3003 & -0.9063 & -6.1332 & 0.3238 \\
-0.9063 & 101.7703 & 0.1872 & -0.0174 \\
-6.1332 & 0.1872 & 100.1997 & 0.3192 \\
0.3238 & -0.0174 & 0.3192 & 102.0761
\end{array}\right] }
\end{aligned}
$$




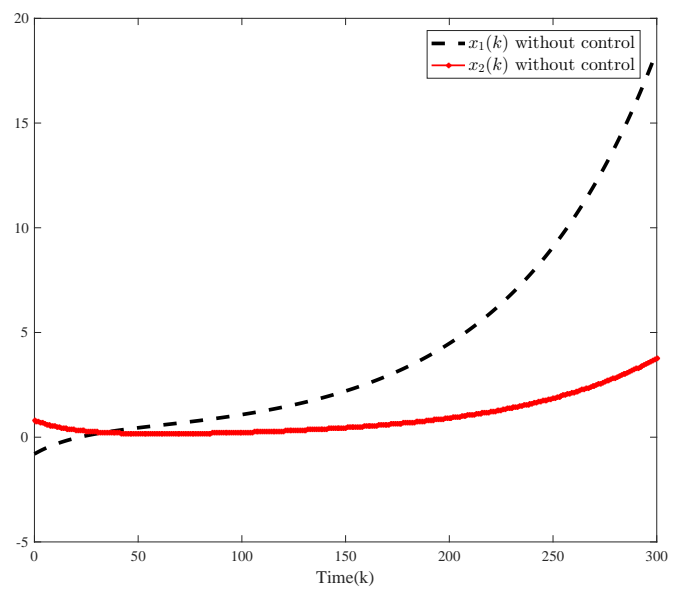

Fig. 1: The evolution of the system state $x(k)$ without control

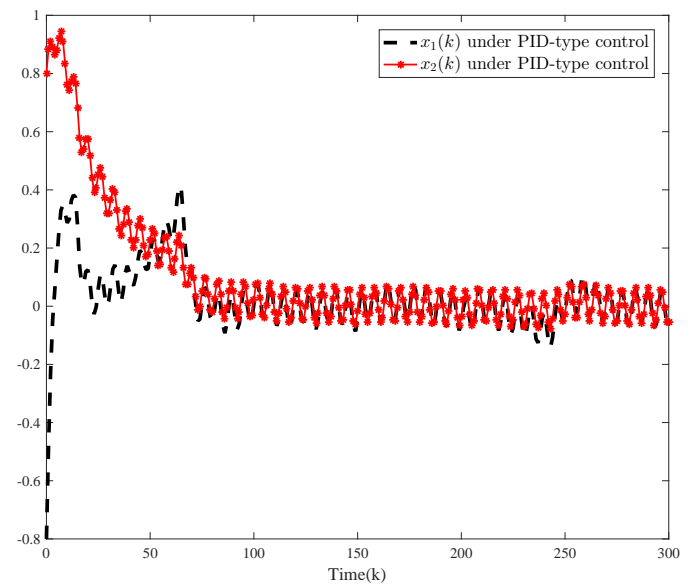

Fig. 2: The evolution of the system state $x(k)$ with PID control

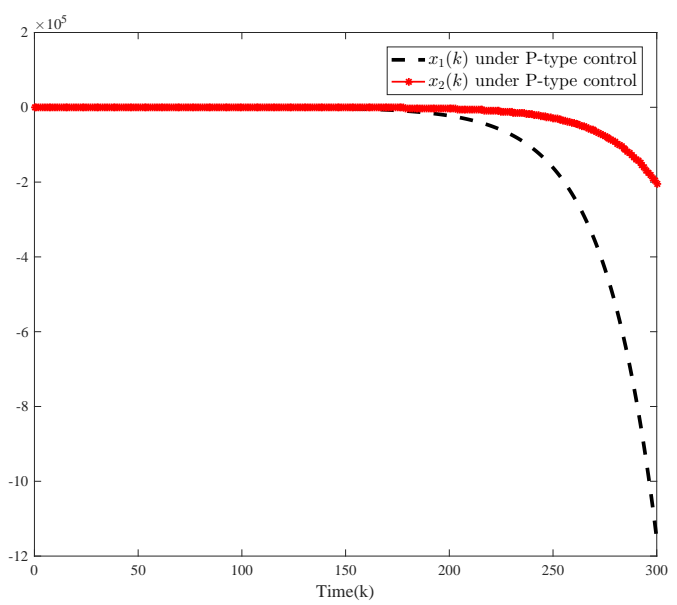

Fig. 3: The evolution of the system state $x(k)$ with proportional control

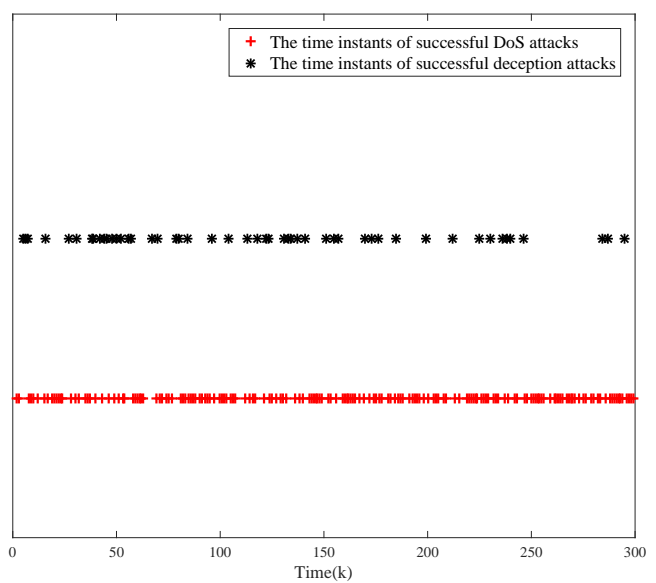

Fig. 4: The time instants of successful DoS/deception attacks

$$
\begin{aligned}
P_{3} & =\left[\begin{array}{cccc}
87.4905 & -2.2146 & -14.0004 & 0.6677 \\
-2.2146 & 91.7392 & 0.6419 & -0.0465 \\
-14.0004 & 0.6419 & 89.5614 & 0.6453 \\
0.6677 & -0.0465 & 0.6453 & 92.4658
\end{array}\right] \\
\hat{M} & =\left[\begin{array}{cc}
106.4406 & -45.0722 \\
-45.0722 & 56.6389
\end{array}\right], \quad \tilde{\kappa}=681.2461 \\
\check{K}_{P} & =\left[\begin{array}{c}
-39.6555 \\
-1.2977
\end{array}\right]^{T}, \quad \check{K}_{I}=\left[\begin{array}{c}
-5.5061 \\
-3.1368
\end{array}\right]^{T} \\
\check{K}_{D} & =\left[\begin{array}{c}
-1.6518 \\
-9.4103
\end{array}\right]^{T}, \Lambda=\left[\begin{array}{cc}
8.7988 & -74.7822 \\
0 & 10.1075
\end{array}\right] .
\end{aligned}
$$

It can be checked that the condition (49) is satisfied. Furthermore, the gain matrices of the observer (8) and the PID controller (9) are outlined as follows:

$$
\begin{array}{rlrl}
L & =\left[\begin{array}{l}
0.4559 \\
0.2521
\end{array}\right], & K_{P} & =\left[\begin{array}{l}
-4.5069 \\
-0.1475
\end{array}\right]^{T} \\
K_{I} & =\left[\begin{array}{l}
-0.6257 \\
-0.3565
\end{array}\right]^{T}, \quad K_{D}=\left[\begin{array}{l}
-0.1877 \\
-1.0694
\end{array}\right]^{T}
\end{array}
$$

and the permitted upper bound $\bar{J}$ is 0.0563 .

The simulation results are presented in Figs. 1-3. Fig. 1 depicts the state trajectory of the system (1) without control which is obviously unstable. In addition, Fig. 2 and Fig. 3 plot the dynamics of the closed-loop system (11), respectively, with the proposed PID-type control and only P-type control. Evidently, the closed-loop system with only the proportional control loop cannot achieve the desired control performance whereas the PID-type one can. The simulation results have confirmed that the observer-based PID control mechanism proposed in this paper is indeed effective.

\section{B. Comparison of Results Between Different Attack Probabil- ities}

In this subsection, some comparison simulations have been conducted to examine the effect from the cyber-attacks on the system performance. The time instants of the successful DoS 
This article has been accepted for publication in a future issue of this journal, but has not been fully edited. Content may change prior to final publication. Citation information: DOI10.1109/TSMC.2019.2952539, IEEE Transactions on Systems, Man, and

TABLE I: THE MINIMUM SECURITY LEVEL WITH DIFFERENT $\bar{\alpha}$ FOR $\bar{\beta}=0.001$

\begin{tabular}{cc}
\hline \hline $\bar{\alpha}$ & Security level $\varrho$ \\
\hline $\bar{\alpha}=0.09$ & 0.0569 \\
$\bar{\alpha}=0.19$ & 0.0866 \\
$\bar{\alpha}=0.29$ & 0.1026 \\
$\bar{\alpha}=0.39$ & 0.1121 \\
$\bar{\alpha}=0.49$ & 0.1341 \\
$\bar{\alpha}=0.59$ & 0.1734 \\
$\bar{\alpha}=0.69$ & 0.2072 \\
$\bar{\alpha}=0.79$ & 0.2382 \\
$\bar{\alpha}=0.89$ & 0.2308 \\
$\bar{\alpha}=0.99$ & 0.2627 \\
\hline \hline
\end{tabular}

TABLE II: THE MINIMUM SECURITY LEVEL WITH DIFFERENT $\bar{\alpha}$ FOR $\bar{\beta}=0.999$

\begin{tabular}{cc}
\hline \hline $\bar{\alpha}$ & Security level $\varrho$ \\
\hline $\bar{\alpha}=0.09$ & 0.0632 \\
$\bar{\alpha}=0.19$ & 0.0974 \\
$\bar{\alpha}=0.29$ & 0.1043 \\
$\bar{\alpha}=0.39$ & 0.1255 \\
$\bar{\alpha}=0.49$ & 0.1451 \\
$\bar{\alpha}=0.59$ & 0.1862 \\
$\bar{\alpha}=0.69$ & 0.1979 \\
$\bar{\alpha}=0.79$ & 0.2135 \\
$\bar{\alpha}=0.89$ & 0.2573 \\
$\bar{\alpha}=0.99$ & 0.2778 \\
\hline \hline
\end{tabular}

attacks and deception attacks are shown in Fig. 4. In Tables III, we also present the minimal security levels (expressed by the bounds of the system states) with the increased attack probability of DoS attacks or deception attacks. It is tempting to conclude from Tables I-II that the security performance deteriorates (the minimal security level increases) as the attack probability increases, which again confirms our results.

\section{CONCLUSION}

In this paper, an observer-based PID control scheme has been developed for a kind of linear discrete-time systems in presence of time-varying delays as well as randomly occurring cyber-attacks. The random nature of the addressed DoS/deception attacks are regulated by two random variables conforming to Bernoulli distribution. A theoretical framework has first been established for the addressed systems to analyze the exponentially mean-square input-to-state stability. Then, within such a framework, sufficient conditions have been put forward to guarantee the prescribed security level and acquire an upper bound on the QCC. Subsequently, the gain parameters of the desired controller have been designed in virtue of the LMI technique combined with orthogonal decomposition. Finally, an illustrative simulation example has been exploited to verify the usefulness of the presented control scheme. Future research themes include the utilization of our methods to more complicated networked control systems subject to more sophisticated network-induced phenomena or cyber-attacks with time-varying probability distributions [23], [35], [48]-[50].

\section{REFERENCES}

[1] K. H. Ang, G. Chong and Y. Li, PID control system analysis, design, and technology, IEEE Transactions on Control Systems Technology, Vol. 3, No. 4, pp. 559-576, 2005.

[2] J. Carvajal, G. Chen and H. Ogmen, Fuzzy PID controller: design performance evaluation and stability analysis, Information Sciences, Vol. 123, No. 3-4, pp. 249-270, 2000.

[3] J. Cecil, S. Albuhamood, A. Cecil-Xavier and P. Ramanathan, An advanced cyber physical framework for micro devices assembly, IEEE Transactions on Systems, Man, and Cybernetics - Systems, Vol. 49, pp. 92-106, 2017.

[4] A. Cetinkaya, H. Ishii and T. Hayakawa, Networked control under random and malicious packet losses, IEEE Transactions on Automatic Control, Vol. 62, No. 5, pp. 2434-2449, 2017.

[5] W. D. Chang and J. J. Yan, Adaptive robust PID controller design based on a sliding mode for uncertain chaotic systems, Chaos, Solitons \& Fractals, Vol. 26, No. 1, pp. 167-175, 2005.

[6] Y. Chen, Z. Wang, W. Qian and F. E. Alsaadi, Asynchronous observerbased $H_{\infty}$ control for switched stochastic systems with mixed delays under quantization and packet dropouts, Nonlinear Analysis: Hybrid Systems, Vol. 27, pp. 225-238, 2018.

[7] Y. Chen, Z. Wang, Y. Yuan and P. Date, Distributed $H_{\infty}$ filtering for switched stochastic delayed systems over sensor networks with fading measurements, IEEE Transactions on Cybernetics, Vol. 50, No. 1, pp. 2-14, Jan. 2020

[8] S. Cong and Y. Liang, PID-like neural network nonlinear adaptive control for uncertain multivariable motion control systems, IEEE Transactions on Industrial Electronics, Vol. 56, No. 10, pp. 3872-3879, 2009.

[9] A. Datta, M. T. Ho and S. P. Bhattacharyya, Structure and synthesis of PID controllers in advances in industrial control, Springer-Verlag, London, U.K, 2000.

[10] D. Ding, Z. Wang, B. Shen and G. Wei, Event-triggered consensus control for discrete-time stochastic multi-agent systems: the input-tostate stability in probability, Automatica, Vol. 62, pp. 284-291, 2015.

[11] D. Ding, Z. Wang, G. Wei and F. E. Alsaadi, Event-based security control for discrete-time stochastic systems, IET Control Theory \& Applications, Vol. 10, No. 15, pp. 1808-1815, 2016.

[12] D. Ding, Z. Wang, D. W. C. Ho and G. Wei, Distributed recursive filtering for stochastic systems under uniform quantizations and deception attacks through sensor networks, Automatica, Vol. 78, pp. 231-240, 2017.

[13] D. Ding, Z. Wang, Q.-L. Han and G. Wei, Security control for discretetime stochastic nonlinear systems subject to deception attacks, IEEE Transactions on Systems, Man, and Cybernetics - Systems, Vol. 48, No. 5, pp. 779-789, 2018.

[14] E. Fridman, M. Dambrine and N. Yeganefar, On input-to-state stability of systems with time-delay: a matrix inequalities approach, Automatica, Vol. 44, No. 9, pp. 2364-2369, 2008.

[15] W. K. Ho, C. C. Hang and L. S. Cao, Tuning of PID controllers based on gain and phase margin specifications, Automatica, Vol. 31, No. 3, pp. 497-502, 1995.

[16] N. Hou, Z. Wang, D. W. C. Ho and H. Dong, Robust partial-nodes-based state estimation for complex networks under deception attacks, IEEE Transactions on Cybernetics, in press, DOI:10.1109/TCYB.2019.2918760.

[17] Z. P. Jiang and Y. Wang, Input-to-state stability for discrete-time nonlinear systems, Automatica, Vol. 37, No. 6, pp. 857-869, 2001.

[18] Z. P. Jiang and Y. Wang, A converse Lyapunov theorem for discretetime systems with disturbances, Systems \& control letters, Vol. 45, No. 1, pp. 49-58, 2002.

[19] D. H. Kim and J. H. Cho, A biologically inspired intelligent PID controller tuning for AVR systems, International Journal of Control, Automation, and Systems, Vol. 4, No. 5, pp. 624-636, 2006.

[20] B. Li, Z. Wang and Q.-L. Han, Input-to-state stabilization of delayed differential systems with exogenous disturbances: the event-triggered case, IEEE Transactions on Systems, Man, and Cybernetics - Systems, in press, DOI: 10.1109/TSMC.2017.2719960, 2018.

[21] J. Li, H. Dong, F. Han, N. Hou and X. Li, Filter design, fault estimation and reliable control for networked time-varying systems: A survey, Systems Science \& Control Engineering, vol. 5, no. 1, pp. 331-341, Jul. 2017.

[22] X. Li, H. Dong, F. Han and J. Li, A survey on set-membership filtering for networked control systems under communication protocols, Systems Science \& Control Engineering, vol. 6, no. 1, pp. 293-303, Jul. 2018. 
[23] S. Liu, Z. Wang, Y. Chen and G. Wei, Protocol-based unscented Kalman filtering in the presence of stochastic uncertainties, IEEE Transactions on Automatic Control, in press, DOI:10.1109/TAC.2019.2929817.

[24] L. Ma, Z. Wang, Q.-L. Han and H. K. Lam, Variance-constrained distributed filtering for time-varying systems with multiplicative noises and deception attacks over sensor networks, IEEE Sensors Journal, Vol. 17, No. 7, pp. 2279-2288, 2017.

[25] E. Mousavinejad, F. Yang, Q.-L. Han and L. Vlacic, A novel cyber attack detection method in networked control systems, IEEE Transactions on Cybernetics, Vol. 48, No. 11, pp. 3254-3264, 2018.

[26] Y. Pan and G.-H. Yang, Event-triggered fault detection filter design for nonlinear networked systems, IEEE Transactions on Systems, Man, and Cybernetics: Systems, Vol. 48, No. 11, pp. 1851-1862, 2017.

[27] Z.-H. Pang, G.-P. Liu, D. Zhou and D. Sun, Data-driven control with input design-based data dropout compensation for networked nonlinear systems, IEEE Transactions on Control Systems Technology, Vol. 25, No. 2, pp. 628-636, 2017

[28] F. Rasool and S. K. Nguang, Robust $H_{\infty}$ state feedback control of NCSs with poisson noise and successive packet dropouts, International Journal of Control, Automation and Systems, Vol. 13, No. 1, pp. 45-57, 2015.

[29] H. Sedjelmaci, S. M. Senouci and N. Ansari, A hierarchical detection and response system to enhance security against lethal cyber-attacks in UAV networks, IEEE Transactions on Systems Man Cybernetics Systems, Vol. 48, No. 9, pp. 1594-1606, 2018.

[30] J. Tavčar and I. Horváth, A review of the principles of designing smart cyber-physical systems for run-time adaptation: learned lessons and open issues, IEEE Transactions on Systems Man Cybernestic-Systems, Vol. 49 , No. 1, pp. 145-158, 2019.

[31] D. Wang, Z. Wang, B. Shen and F. E. Alsaadi, Security-guaranteed filtering for discrete-time stochastic delayed systems with randomly occurring sensor saturations and deception attacks, International Journal of Robust and Nonlinear Control, Vol. 27, No. 7, pp. 1194-1208, 2017.

[32] L. Wang, Z. Wang, G. Wei and F. E. Alsaadi, Observer-based consensus control for discrete-time multi-agent systems with coding-decoding communication protocol, IEEE Transactions on Cybernetics, in press, DOI:10.1109/TCYB.2018.2863664

[33] L. Wang, Z. Wang, Q.-L. Han and G. Wei, Event-based varianceconstrained $H_{\infty}$ filtering for stochastic parameter systems over sensor networks with successive missing measurements, IEEE Transactions on Cybernetics, vol. 48, no. 3, pp. 1007-1017, Mar. 2018.

[34] M. Wang, Z. Wang, Y. Chen and W. Sheng, Adaptive neural event-triggered control for discrete-time strict-feedback nonlinear systems, IEEE Transactions on Cybernetics, in press, DOI:10.1109/TCYB.2019.2921733.

[35] M. Wang, Z. Wang, Y. Chen and W. Sheng, Observer-based fuzzy output-feedback control for discrete-time strict-feedback nonlinear systems with stochastic noises, IEEE Transactions on Cybernetics, in press, DOI:10.1109/TCYB.2019.2902520.

[36] Z. Wang, J. Lam, L. Ma, Y. Bo and Z. Guo, Variance-constrained dissipative observer-based control for a class of nonlinear stochastic systems with degraded measurements, Journal of Mathematical Analysis and Applications, Vol. 377, No. 2, pp. 645-658, 2011.

[37] Z. Wang, L. Wang, S. Liu and G. Wei, Encoding-Decoding-Based control and filtering of networked systems: insights, developments and opportunities, IEEE/CAA Journal of Automatica Sinica, Vol. 5, No. 1, pp. 3-18, 2018.

[38] W. Xu, Z. Wang and D. W. C. Ho, Finite-horizon $H_{\infty}$ consensus for multiagent systems with redundant channels via an observer-type eventtriggered scheme, IEEE Transactions on Cybernetics, Vol. 48, No. 5 , pp. 1567-1576, May 2018.

[39] Y. Yuan, Z. Wang, P. Zhang and H. Liu, Near-optimal resilient control strategy design for state-saturated networked systems under stochastic communication protocol, IEEE Transactions on Cybernetics, vol. 49, no. 8, pp. 3155-3167, Aug. 2019.

[40] H. Zhang, Y. Shi and A. S. Mehr, Robust static output feedback control and remote PID design for networked motor systems, IEEE Transactions on Industrial Electronics, Vol. 58, No. 12, pp. 5396-5405, 2011.

[41] S. Zhang, Z. Wang, D. Ding, G. Wei, F. E. Alsaadi and T. Hayat, A gain-scheduling approach to nonfragile $H_{\infty}$ fuzzy control subject to fading channels, IEEE Transactions on Fuzzy Systems, Vol. 26, No. 1, pp. 142-154, 2018.

[42] X.-M. Zhang, Q.-L. Han and X. Yu, Survey on recent advances in networked control systems, IEEE Transactions on Industrial Informatics, Vol. 12, No. 5, pp. 1740-1752, 2016.

[43] X.-M. Zhang, Q.-L. Han and B.-L. Zhang, An overview and deep investigation on sampled-data-based event-triggered control and filtering for networked systems, IEEE Transactions on Industrial Informatics, Vol. 13, No. 1, pp. 4-16, 2017.

[44] W. Zhou, L. Teng and D. Xu, Mean-square exponentially input-tostate stability of stochastic Cohen-Grossberg neural networks with timevarying delays, Neurocomputing, Vol. 153, pp. 54-61, 2015.

[45] M. Zhu and S. Martínez, On the performance analysis of resilient networked control systems under replay attacks, IEEE Transactions on Automatic Control, Vol. 59, No. 3, pp. 804-808, 2014.

[46] J. G. Ziegler and N. B. Nichols, Optimum settings for automatic controllers, ASME Transactions, Vol. 64, No. 11, 1942.

[47] Z. Zhao, Z. Wang, L. Zou and G. Guo, Finite-time state estimation for delayed neural networks with redundant delayed channels, IEEE Transactions on Systems, Man, and Cybernetics-Systems, in press, DOI:10.1109/TSMC.2018.2874508.

[48] L. Zou, Z. Wang, Q.-L. Han and D. H. Zhou, Full information estimation for time-varying systems with Round-Robin protocol: A recursive filter approach, IEEE Transactions on Systems, Man, and CyberneticsSystems, in press, DOI:10.1109/TSMC.2019.2907620.

[49] L. Zou, Z. Wang, Q.-L. Han and D. H. Zhou, Moving horizon estimation of networked nonlinear systems with random access protocol, IEEE Transactions on Systems, Man, and Cybernetics-Systems, in press, DOI:10.1109/TSMC.2019.2918002.

[50] L. Zou, Z. Wang, Q.-L. Han and D. Zhou, Moving horizon estimation for networked time-delay systems under RoundRobin protocol, IEEE Transactions on Automatic Control, in press, DOI:10.1109/TAC.2019.2910167.

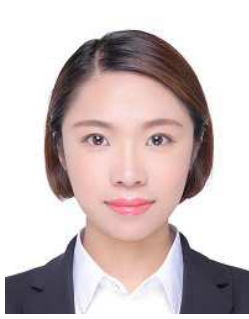

Di Zhao received the B.Sc. degree in Mathematics from Jilin Normal University, Siping, China, in 2013. She is currently working toward the Ph.D. degree in Control Science and Engineering from University of Shanghai for Science and Technology, Shanghai, China.

From November 2018 to November 2019, she was a visiting Ph.D. student in the Department of Computer Science, Brunel University London, Uxbridge, U.K.. From March to June 2018, she was a Research Associate in the Department of Mathematics, City University of Hong Kong, Hong Kong.

Her research interests include PID control, proportional-integral observer, networked control systems and cyber-physical systems. She is currently a reviewer for some international journals.

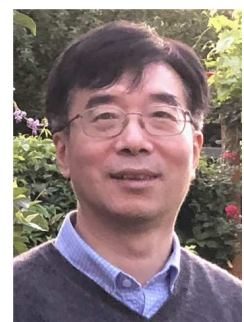

Zidong Wang (SM'03-F'14) was born in Jiangsu, China, in 1966. He received the B.Sc. degree in mathematics in 1986 from Suzhou University, Suzhou, China, and the M.Sc. degree in applied mathematics in 1990 and the Ph.D. degree in electrical engineering in 1994, both from Nanjing University of Science and Technology, Nanjing, China.

$\mathrm{He}$ is currently a Professor of Dynamical Systems and Computing in the Department of Computer Science, Brunel University London, UK. From 1990 to 2002 , he held teaching and research appointments in universities in China, Germany and the UK. His research interests include dynamical systems, signal processing, bioinformatics, control theory and applications. He has published 220+ papers in IEEE Transactions and 60+ papers in Automatica. He is a holder of the Alexander von Humboldt Research Fellowship of Germany, the JSPS Research Fellowship of Japan, William Mong Visiting Research Fellowship of Hong Kong.

Prof. Wang serves (or has served) as the Editor-in-Chief for Neurocomputing, the Deputy Editor-in-Chief for International Journal of Systems Science, and an Associate Editor for 12 international journals including IEEE Transactions on Automatic Control, IEEE Transactions on Control System Technology, IEEE Transactions on Neural Networks, IEEE Transactions on Signal Processing, and IEEE Transactions on Systems, Man, and CyberneticsSystems. He is a Fellow of the IEEE, a Fellow of the Royal Statistical Society and a member of program committee for many international conferences. 


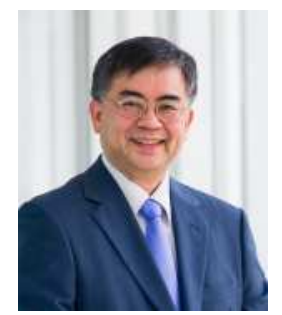

Daniel W.C. Ho (SM'05-F'17) received BSc, MSc and $\mathrm{PhD}$ degrees in mathematics from the University of Salford (UK) in 1980, 1982 and 1986 respectively. From 1985 to 1988 , he was a Research Fellow in Industrial Control Unit, University of Strathclyde (Glasgow, Scotland). In 1989, he joined the Department of Mathematics, City University of Hong Kong. Currently, he is the Chair Professor and Assistant Dean in Teaching Innovations of College of Science and Engineering.

Prof. Ho serves as Subject editor for Journal of the Franklin Institute, Associate Editor for five other international Control journals including IET Control Theory and its applications, and Asian Journal of Control. He is recipient of "The Best paper award" from the 8th Asian Control Conference. In 2012, he is honored to be the Chang Jiang Chair Professor, awarded by Ministry of Education, China. Recently, he is named as Highly Cited Researchers in Engineering by Thomson Reuters in 2014 and 2015.

From 1985 to 1988, he was a Research Fellow with the Industrial Control Unit, University of Strathclyde, Glasgow, U.K. In 1989, he joined the College of Science and Engineering, City University of Hong Kong, Hong Kong, as the Assistant Dean (Teaching Innovations), where he is currently a Chair Professor in Applied Mathematics with the Department of Mathematics. He has published over 200 publications in scientific journals. His current research interests include control and estimation theory, complex dynamical distributed networks, multi-agent networks, and stochastic systems.

Dr. Ho was a recipient of the Chang Jiang Chair Professor Award by the Ministry of Education, China, in 2012 and the ISI Highly Cited Researchers Award in Engineering by Thomson Reuters, from 2014 to 2016. He has been on the Editorial Board of a number of journals including the IEEE TRANSACTIONS ON NEURAL NETWORKS AND LEARNING SYSTEMS, IET Control Theory and Its Applications, the Journal of the Franklin Institute, and the Asian Journal of Control.

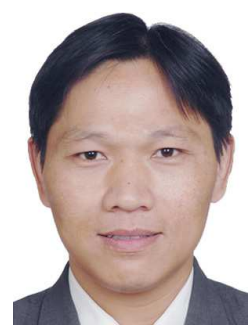

Guoliang Wei received the B.Sc. degree in mathematics from Henan Normal University, Xinxiang, China, in 1997 and the M.Sc. degree in applied mathematics and the Ph.D. degree in control engineering, both from Donghua University, Shanghai, China, in 2005 and 2008, respectively. He is currently a Professor with the Department of Control Science and Engineering, University of Shanghai for Science and Technology, Shanghai, China.

From March 2010 to May 2011, he was an Alexander von Humboldt Research Fellow in the Institute for Automatic Control and Complex Systems, University of DuisburgEssen, Germany. From March 2009 to February 2010, he was a post doctoral research fellow in the Department of Information Systems and Computing, Brunel University, Uxbridge, U.K., sponsored by the Leverhulme Trust of the U.K.. From June to August 2007, he was a Research Assistant at the University of Hong Kong. From March to May 2008, he was a Research Assistant at the City University of Hong Kong.

His research interests include nonlinear systems, stochastic systems, and bioinformatics. He has published more than 100 papers in refereed international journals. He is a very active reviewer for many international journals. 